\title{
A catalog of extended clusters and ultra-compact dwarf galaxies
}

\section{An analysis of their parameters in early- and late-type galaxies ${ }^{\star} \star \star$}

\author{
R. C. Brüns and P. Kroupa
}

\author{
Argelander-Institut für Astronomie, Universität Bonn, Auf dem Hügel 71, 53121 Bonn, Germany \\ e-mail: [rcbruens; pavel]@astro.uni-bonn.de
}

Received 28 May 2012 / Accepted 14 September 2012

\section{ABSTRACT}

\begin{abstract}
Context. In the past decade, very extended old stellar clusters with masses in the range from a few $10^{4}$ to $10^{8} M_{\odot}$ and effective radii larger than $10 \mathrm{pc}$ have been found in various types of galaxies in different environments. Objects with masses comparable to normal globular clusters (GCs) are called extended clusters (ECs), while objects with masses in the dwarf galaxy regime are called ultra-compact dwarf galaxies (UCDs).

Aims. The paper analyses the observational parameters total luminosity, $M_{\mathrm{V}}$, effective radius, $r_{\mathrm{eff}}$, and projected distance to the host galaxy, $R_{\text {proj }}$, of all known ECs and UCDs and the dependence of these parameters on the type and the total luminosity of their host galaxy.

Methods. We searched the available literature to compile a catalog of star clusters with effective radii larger than $10 \mathrm{pc}$. As there is no clear distinction between ECs and UCDs, both types of objects will be called extended stellar objects - abbreviated "EOs" - in this paper.

Results. In total, we found 813 EOs of which 171 are associated with late-type galaxies and 642 EOs associated with early-type galaxies. EOs cover a luminosity range from about $M_{\mathrm{V}}=-4$ to -14 mag. However, the vast majority of EOs brighter than $M_{\mathrm{V}}=$ $-10 \mathrm{mag}$ are associated with giant elliptical galaxies. At each magnitude extended objects are found with effective radii between $10 \mathrm{pc}$ and an upper size limit, which shows a clear trend: the more luminous the object the larger is the upper size limit. For EOs associated with early- and late-type galaxies, the EO luminosity functions peak at -6.40 mag and -6.47 mag, respectively, which is about one magnitude fainter than the peak of the GC luminosity function. EOs and GCs form a coherent structure in the $r_{\text {eff }}$ vs. $M_{\mathrm{V}}$ parameter space, while there is a clear gap between EOs and early-type dwarf galaxies. However, there is a small potential overlap at the high-mass end, where the most extended EOs are close to the parameters of some compact elliptical galaxies. We compare the EO sample with the numerical models of a previous paper and conclude that the parameters of the EO sample as a whole can be very well explained by a star cluster origin, where EOs are the results of merged star clusters of cluster complexes.
\end{abstract}

Key words. catalogs - galaxies: star clusters: general

\section{Introduction}

Globular clusters (GCs) are typically very old stellar objects with masses between $10^{4} M_{\odot}$ and $10^{6} M_{\odot}$ (corresponding roughly to total luminosities between $M_{\mathrm{V}}=-5$ to $M_{\mathrm{V}}=-10 \mathrm{mag}$ ), having in general compact sizes with half-light radii of a few parsecs. This morphology makes them easily observable also in external galaxies with modern telescopes (see Brodie \& Strader 2006, and references therein).

The Milky Way has a rich GC system containing 157 GCs (Harris 1996, 2010 edn.). Most of them are compact with sizes of a few parsec. Only $13 \mathrm{GCs}$ (or $8 \%$ ) have an effective radius larger than $10 \mathrm{pc}$. Most of these extended clusters (ECs) are fainter than $M_{\mathrm{V}}=-7 \mathrm{mag}$, only NGC 2419 , having a half-light radius of about $20 \mathrm{pc}$, has a high luminosity of about $M_{\mathrm{V}}=-9.4 \mathrm{mag}$. Two of the 13 ECs, Arp2 and Terzan8, were classified as Milky Way clusters in Harris (1996, 2010 edn.), but the proximity to the Sagittarius dwarf spheriodal galaxy suggests that they are related to this Milky Way satellite (Salinas et al. 2012). Further ECs in the vicinity of the Milky Way have been found in the

\footnotetext{
* Table 2 is available in electronic form at http: //www . aanda. org

$\star \star$ Table 1 is only available at the CDS via anonymous ftp to cdsarc.u-strasbg.fr (130.79.128.5) or via

http://cdsarc.u-strasbg.fr/viz-bin/qcat? J/A+A/547/A65
}

LMC and the Fornax dwarf galaxy (Mackey \& Gilmore 2004; van den Bergh \& Mackey 2004; McLaughlin \& van der Marel 2005).

Comparable objects have also been detected around other galaxies. Huxor et al. (2005) found three ECs around M31, which have very large radii above 30 pc. These clusters were detected by chance as the automatic detection algorithms of the MegaCam Survey discarded such extended objects as likely background contaminations. Further observations increased the number of ECs in M31 to 13 (Huxor et al. 2008). However, Huxor et al. (2011a) showed that the previous estimates of the effective radii were considerably too large. The new size estimates are well below $30 \mathrm{pc}$.

In addition to ECs located in galactic halos, Larsen \& Brodie (2000) and Brodie \& Larsen (2002) have discovered a population of ECs co-rotating with the disk of the lenticular galaxy NGC 1023. These so-called faint fuzzies have similar structural parameters as halo ECs.

Chandar et al. (2004) observed a part of the disks of the nearby spiral galaxies M 81, M 83, NGC 6946, M 101, and M 51 using Hubble Space Telescope (HST) and found ECs with effective radii larger than $10 \mathrm{pc}$ in four of them. In recent years, hundreds of ECs have been detected in all types of galaxies ranging from dwarfs to giant elliptical galaxies. 
Hilker et al. (1999) and Drinkwater et al. (2000) discovered in the Fornax Cluster compact objects with luminosities above the brightest known GCs and which were not resolved by ground-based observations. These objects have masses between a few $10^{6} M_{\odot}$ and $10^{8} M_{\odot}$ and effective radii between $r_{\text {eff }}=10$ and 100 pc. Phillipps et al. (2001) interpreted these objects as a new type of galaxy and reflected this notion in the name "ultracompact dwarf galaxy" (UCD). Next to the Fornax Cluster, many UCDs have been found also in other clusters like the Virgo Cluster (Haşegan et al. 2005; Evstigneeva et al. 2007), the Centaurus Cluster (Mieske et al. 2007), the Coma Cluster (Madrid et al. 2010), and the Hydra Cluster (Misgeld et al. 2011). While most known UCDs belong to elliptical galaxies in cluster environments, they have also been observed in rather isolated objects like the Sombrero galaxy M 104 (Hau et al. 2009) or the group elliptical NGC 3923 (Norris \& Kannappan 2011).

A number of different origins of UCDs were brought up next to the original proposal of UCDs being just a new type of galaxy (Phillipps et al. 2001). Bekki et al. (2001, 2003) suggested that UCDs are the remnants of dwarf galaxies which lost their dark matter halo and all stars except for their nucleus. Next to the interpretation as a galaxy, UCDs were also considered as highmass versions of normal GCs (Mieske et al. 2002), or as merged massive complexes of star clusters (Kroupa 1998; Fellhauer \& Kroupa 2002a; Bekki et al. 2004). Forbes et al. (2008) and Mieske et al. (2008) analyzed the parameters of UCDs and concluded that UCDs are more likely bright extended GCs than naked cores of stripped dwarf galaxies. The marginally enhanced mass-to-light ratios of UCDs can be explained by slightly modified initial stellar mass functions (Mieske \& Kroupa 2008; Dabringhausen et al. 2009).

High-resolution HST imaging of gas-rich galaxies experiencing major interactions has resolved very intense star formation bursts. Bastian et al. (2006a) observed star cluster complexes (CCs), i.e. clusters of young massive star clusters, in the Antennae with masses of the order $\approx 10^{6} M_{\odot}$ and diameters of the order 100 to $200 \mathrm{pc}$. Whitmore et al. (2005) found that the cluster to cluster velocity dispersion in some CCs in the Antennae, aka the knots, is small enough to keep them gravitationally bound. Pellerin et al. (2010) detected young massive CCs with masses between $10^{6} M_{\odot}$ and $10^{7.5} M_{\odot}$ and diameters between $600 \mathrm{pc}$ and $1200 \mathrm{pc}$ in the collisional ring galaxy NGC 922. One of the most extended CCs has been observed by Tran et al. (2003) in the tail of the "Tadpole galaxy" UGC 10214. This CC, which has a mass of the order $10^{6} M_{\odot}$, has an effective radius of $160 \mathrm{pc}$ and a diameter of about $1500 \mathrm{pc}$. In a previous paper, we proposed a common origin of ECs and UCDs based on the merged star cluster scenario (Brüns et al. 2011). We performed a parametric study that systematically scanned a suitable parameter space of CCs and performed numerical simulations to study their further evolution. We concluded that the observed ECs and UCDs can be well explained as evolved CCs.

In recent years, the number of observed ECs and UCDs has rapidly increased for all types of galaxies in various environments. As there is no clear distinction between ECs and UCDs, both types of objects will be called extended stellar objects abbreviated "EOs" - in this paper. The high number of EOs known today, allows for the first time for a detailed analysis of the properties of EOs to disclose commonalities and distinctions of objects from early- and late-type galaxies.

The paper is structured as follows: in Sect. 2 we compile a catalog of ECs and UCDs on the basis of the available publications containing structural parameters of ECs and UCDs.
Table 1. Catalog of the 813 EOs presented in this paper.

\begin{tabular}{lllcccc}
\hline \hline Name & Alt. name & $\begin{array}{c}r_{\text {eff }} \\
(\mathrm{pc})\end{array}$ & $\begin{array}{c}M_{\mathrm{V}} \\
(\mathrm{mag})\end{array}$ & $\begin{array}{c}R_{\text {proj }} \\
(\mathrm{kpc})\end{array}$ & Ref. \\
\hline 1 & MilkyWayEO-01 & NGC 2419 & 21.4 & -9.42 & 89.9 & 1 \\
2 & MilkyWayEO-02 & NGC 5466 & 10.7 & -6.98 & 16.3 & 1 \\
3 & MilkyWayEO-03 & NGC 5053 & 13.2 & -6.76 & 17.8 & 1 \\
$\ldots$ & & & & & & \\
\hline
\end{tabular}

Notes. This table is available in its entirety in a machine-readable form at the CDS. A portion is shown here for guidance regarding its form and content. The columns denote 1 . running number, 2 . designation of EO in this paper, 3. alternative name or designation in original paper, 4. effective radius of the EO, 5. absolute $V$-band luminosity of the EO, 6. projected distance to the host galaxy (for the Milky Way the galactocentric distance is used).

References. (1) Harris (1996); (2) Salinas et al. (2012); (3) van den Bergh \& Mackey (2004); (4) Hwang et al. (2011); (5) Huxor et al. (2008); (6) Huxor et al. (2011a); (7) Peacock et al. (2009); (8) Stonkutè et al. (2008); (9) Cockcroft et al. (2011); (10) Georgiev et al. (2009); (11) Taylor et al. (2010); (12) Gomez et al. (2006); (13) McLaughlin et al. (2008); (14) Mouhcine et al. (2010); (15) Chattopadhyay et al. (2009); (16) Nantais et al. (2011); (17) Strader et al. (2012); (18) Sharina et al. (2005); (19) van den Bergh (2006); (20) Da Costa et al. (2009); (21) Chandar et al. (2004); (22) Hwang \& Lee (2008); (23) Hau et al. (2009); (24) Larsen et al. (2001); (25) Harris et al. (2009); (26) Larsen \& Brodie (2000); (27) Norris \& Kannappan (2011); (28) Chies-Santos et al. (2011); (29) Haşegan et al. (2005); (30) Chilingarian \& Mamon (2008); (31) Evstigneeva et al. (2008); (32) Brodie et al. (2011); (33) Evstigneeva et al. (2007); (34) Chies-Santos et al. (2007); (35) Mieske et al. (2008); (36) Hilker et al. (2007); (37) Richtler et al. (2005); (38) Chilingarian et al. (2011); (39) DeGraaf et al. (2007); (40) Goudfrooij (2012); (41) Blom et al. (2012); (42) Chies-Santos et al. (2006); (43) Cantiello et al. (2009); (44) Da Rocha et al. (2011); (45) Mieske et al. (2007); (46) Misgeld et al. (2011); (47) Penny et al. (2012); (48) Chiboucas et al. (2011); (49) Madrid et al. (2010); (50) Madrid (2011); (51) Blakeslee \& Barber (2008).

In Sect. 3 we present the results of the catalog that are discussed in Sect. 4. Section 5 provides a summary and conclusions.

\section{Observational basis}

As already indicated in the previous section, GC-like objects with effective radii above $10 \mathrm{pc}$, which cover a large luminosity range, have been found in various environments from dwarf to giant elliptical galaxies. To allow for an analysis of their parameters, we compiled a catalog of effective radii, absolute $V$-band luminosities, and projected distances of EOs to their host galaxies as well as the absolute $V$-band luminosities of these galaxies and their distance to the Milky Way. We distinguish between EOs found in late-type galaxies, i.e. spiral and irregular galaxies, and early-type galaxies, i.e. elliptical, lenticular, and dwarf spheroidal galaxies. In Table 1, available at the CDS, we present the catalog of the 813 EOs used in this study. Table 2 provides an overview on the galaxies, where EOs were detected, the number of EOs per galaxy and the luminosity range of the detected EOs.

\subsection{EOs in late-type galaxies}

According to the 2010 edition of the GC catalog of Harris (1996) and considering that Arp2 and Terzan8 are associated with the Sagittarius dwarf spheriodal galaxy (Salinas et al. 2012), the Milky Way has 11 EOs. The other two Local Group spiral 
galaxies M 31 and M 33 have 20 (Huxor et al. 2008; Peacock et al. 2009; Huxor et al. 2011a) and 2 (Stonkutė et al. 2008; Cockcroft et al. 2011) EOs, respectively. EOs were also found in the LMC (van den Bergh \& Mackey 2004), and the dwarf irregular galaxy NGC 6822 (Hwang et al. 2011).

Outside the Local Group, EOs were detected in the spiral galaxies M 81 (Chandar et al. 2004; Nantais et al. 2011), M 83 (Chandar et al. 2004), M 51 (Chandar et al. 2004; Hwang \& Lee 2008), NGC 891 (Harris et al. 2009), NGC 3370 (Cantiello et al. 2009), and in the Sombrero Galaxy M 104 (Larsen et al. 2001; Hau et al. 2009). In addition, EOs were found in the dwarf irregular and the magellanic type dwarf galaxies UGCA 86, UGC 8638, NGC 247, NGC 5237, ESO269-58, NGC 784, NGC 4605, UGC 3974, and NGC 1311 (Georgiev et al. 2009), UGC 7605, KK 065, UGC 3755, KK 112, and UGC 4115 (Sharina et al. 2005; van den Bergh 2006), and NGC 4449 (Strader et al. 2012).

In total, the EO catalog contains 171 EOs associated with late-type galaxies.

\subsection{EOs in early-type galaxies}

EOs were detected in a large number of elliptical galaxies: NGC 5128 (Gomez et al. 2006; McLaughlin et al. 2008; Chattopadhyay et al. 2009; Taylor et al. 2010; Mouhcine et al. 2010), NGC 4660 (Chies-Santos et al. 2011), IC 3652 (Haşegan et al. 2005), NGC 4278 (Chies-Santos et al. 2011), NGC 4486B (Haşegan et al. 2005), M 89 (Haşegan et al. 2005; Chies-Santos et al. 2011), M 59 (Chilingarian \& Mamon 2008), M 49 (Haşegan et al. 2005), M 86 (Chies-Santos et al. 2011), the central galaxy of the Virgo Cluster M87 (Haşegan et al. 2005; Evstigneeva et al. 2008; Brodie et al. 2011; Chies-Santos et al. 2011), M 84 (Chies-Santos et al. 2011), the central galaxy of the Fornax Cluster NGC 1399 (Richtler et al. 2005; Evstigneeva et al. 2007; Hilker et al. 2007; Evstigneeva et al. 2008; Mieske et al. 2008; Chilingarian et al. 2011), NGC 3923 (Norris \& Kannappan 2011), NGC 4476 (Haşegan et al. 2005), NGC 5846 (Chies-Santos et al. 2006), NGC 4696 (Mieske et al. 2007), NGC 3311 (Misgeld et al. 2011), IC 4041, NGC 4889, IC 3998, IC 4030, IC 4041, and NGC 4908 (Chiboucas et al. 2011), NGC 4874 (Madrid et al. 2010; Chiboucas et al. 2011), NGC 1132 (Madrid 2011), NGC 1275 (Penny et al. 2012), NGC 4365 (Blom et al. 2012), NGC 1316 (Goudfrooij 2012), NGC 1199 (Da Rocha et al. 2011), and ESO325-G004 (Blakeslee \& Barber 2008).

In five lenticular galaxies, EOs were detected: in NGC 1023 (Larsen \& Brodie 2000; Brodie \& Larsen 2002) and NGC 1380 (Chies-Santos et al. 2007), 15 and 13 EOs were found, respectively. One EO was discovered in NGC 4546 (Norris \& Kannappan 2011), three EOs in NGC 1533 (DeGraaf et al. 2007), and four EOs were found in M 85 (Haşegan et al. 2005; Chies-Santos et al. 2011).

The EO of the dwarf elliptical Scl-dE1 (Da Costa et al. 2009), the two EOs of the Sagittarius dSph galaxy (Salinas et al. 2012), the EO of the dSph galaxy Fornax (van den Bergh \& Mackey 2004), the 6 EOs of the dSph galaxy KK 84 (Sharina et al. 2005; van den Bergh 2006), and the EO of the dSph galaxy IKN (Georgiev et al. 2009) are contributing to the list of EOs associated with early-type galaxies.

In total, 642 EOs were found in early-type galaxies, 595 thereof are associated with elliptical galaxies. The EO sample of the elliptical galaxies is dominated by the two galaxies NGC 4365 and NGC 1275, which have 217 and 84 EO candidates, respectively.

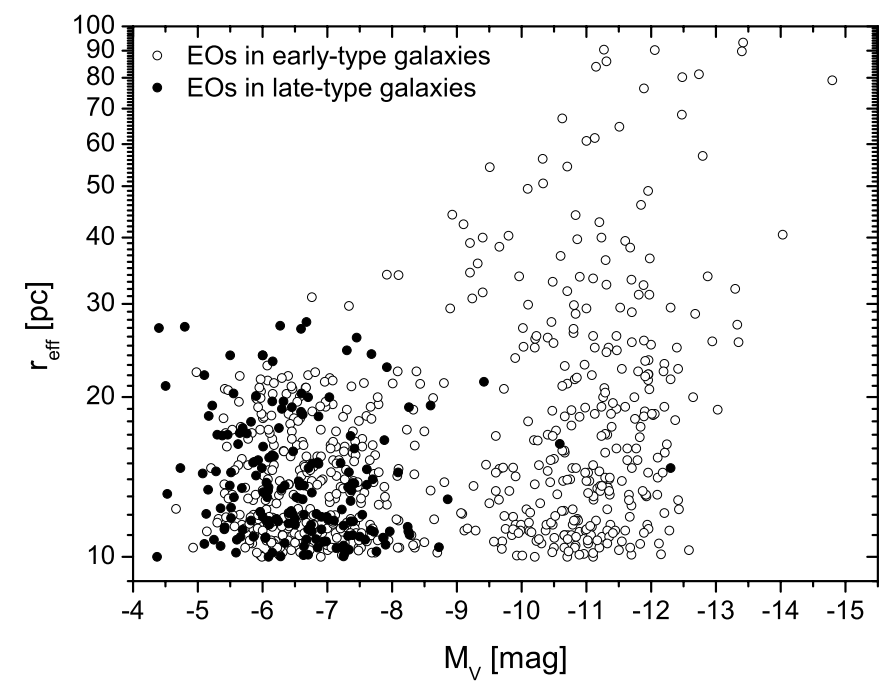

Fig. 1. Effective radii of EOs are plotted against their absolute $V$-band luminosities. EOs associated with early-type galaxies are plotted as open circles, EOs associated with late-type galaxies are plotted as black circles.

\section{Results}

\subsection{Correlations between the parameters of extended objects}

Figure 1 shows the effective radii $r_{\text {eff }}$ of the 813 EOs of our cata$\log$ as a function of their total $V$-band luminosities $M_{\mathrm{V}}$. The vast majority of EOs associated with late-type galaxies have magnitudes between $M_{\mathrm{V}}=-4$ to $-9 \mathrm{mag}$. Only three EOs, the Milky Way cluster NGC 2419 and two EOs associated with the Sombrero Galaxy M 104, have total $V$-band luminosities brighter than $M_{\mathrm{V}}=-9$ mag. EOs associated with early-type galaxies cover a significantly larger range of $V$-band luminosities. The majority of objects are found in the magnitude range of about $M_{\mathrm{V}}=-5$ to $-13 \mathrm{mag}$. At $M_{\mathrm{V}}=-8.5 \mathrm{mag}$ the number of objects is much smaller than for lower and higher luminosities.

At each magnitude EOs are found with effective radii between $10 \mathrm{pc}$ and an upper size limit, which shows a clear trend: the more luminous the object the larger is the upper size limit.

The dependency of the structural parameters effective radius, $r_{\mathrm{eff}}$, and luminosity, $M_{\mathrm{V}}$, of EOs on the projected distance from their host galaxy, $R_{\text {proj }}$, are displayed in Fig. 2. For latetype galaxies there is a slight trend of increasing effective radii and decreasing total luminosities with increasing projected distance (Figs. 2a and 2c). On the other hand, very faint and very extended objects are extremely hard to detect at very low projected distances due to the high surface brightness of the underlying host galaxy. Consequently, the slight trends might not be significant. In early-type galaxies the most extended objects are found predominantly at large projected distances (Fig. 2b). The most extended EO, VUCD7, with an effective radius of $93.2 \mathrm{pc}$, was discovered in the central elliptical galaxy M 87 of the Virgo Cluster at a projected distance of $82.6 \mathrm{kpc}$. In early-type galaxies, the low-luminosity objects are comparable to those in latetype galaxies and there are high-luminosity objects that are not present in late-type galaxies. The void at projected distances larger than about $70 \mathrm{kpc}$ and luminosities fainter than $-10.5 \mathrm{mag}$ is a result of the limited coverage and sensitivity of most surveys.

Figure 3 shows two histograms of the number of EOs with different total $V$-magnitudes. The EOs in late-type galaxies have a luminosity distribution which peaks at around $M_{\mathrm{V}}=-6.5 \mathrm{mag}$, 


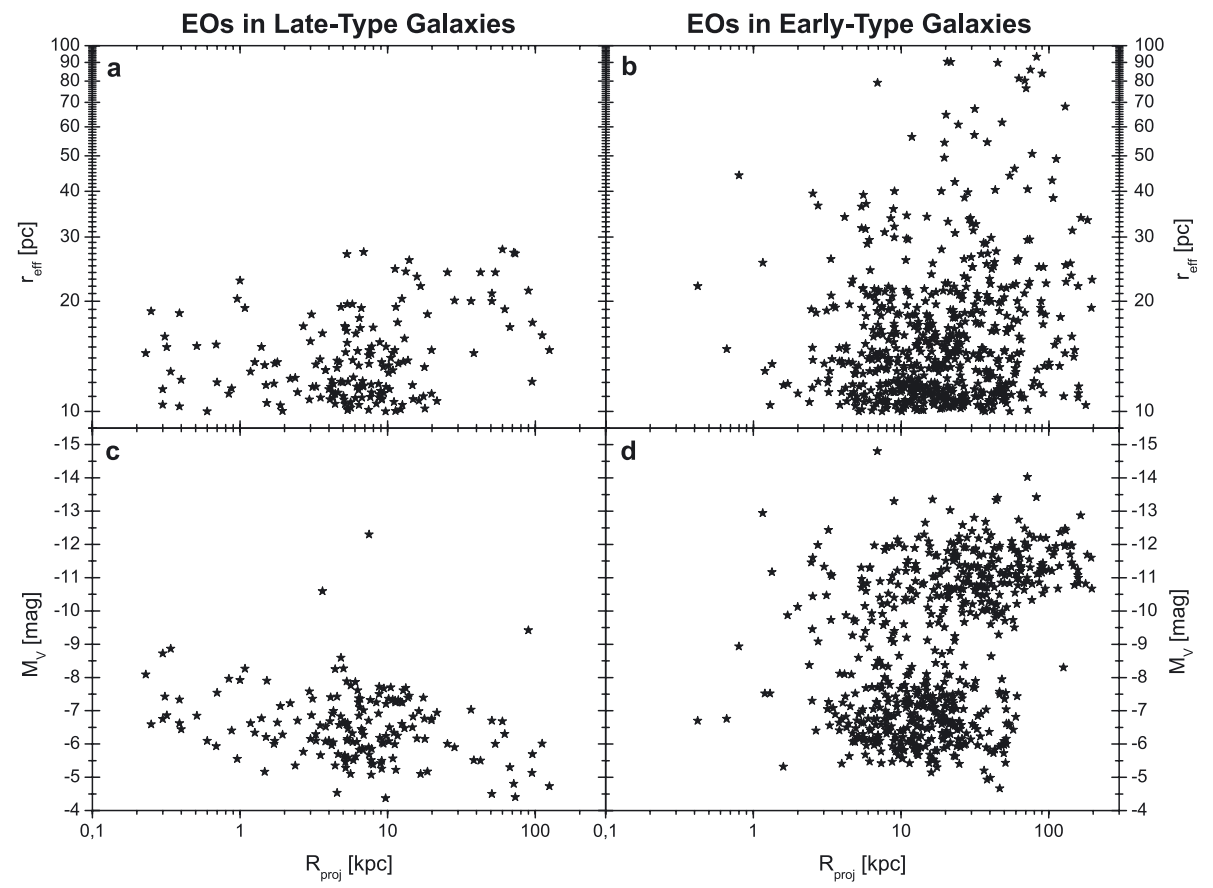

Fig. 2. a) and b) Effective radii of EOs are plotted against the projected distances to their host galaxies. c) and d) Absolute $V$-magnitudes of EOs are plotted against the projected distance.

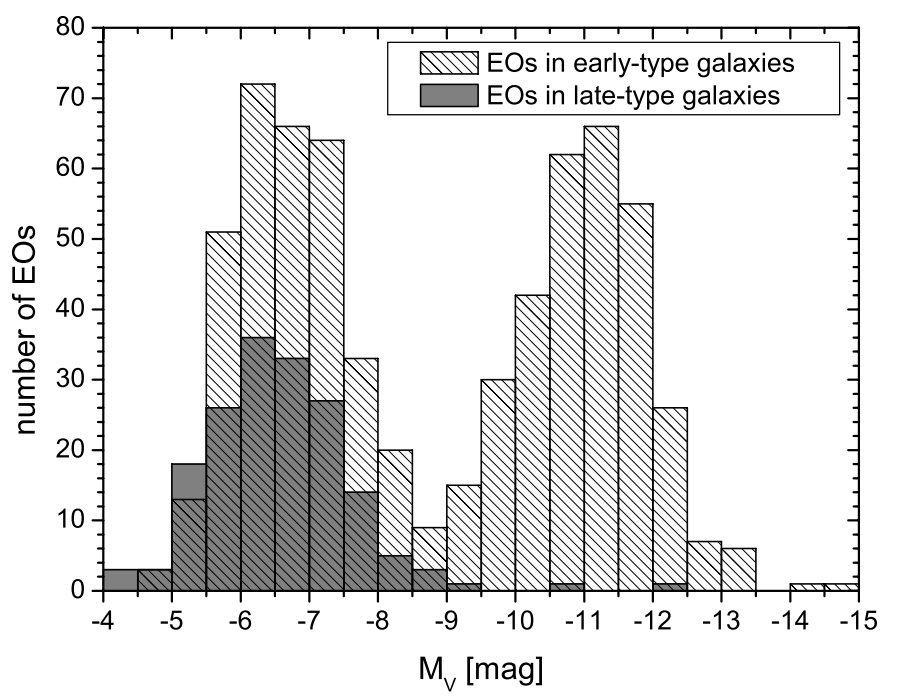

Fig. 3. Histogram of the number of EOs in early-type and late-type galaxies at different total $V$-band luminosities.

while the EOs in early-type galaxies show a bimodal distribution which peaks at $M_{\mathrm{V}}=-6.5 \mathrm{mag}$ and $-11.0 \mathrm{mag}$ and has a clear minimum between -8.5 and -9 mag. In Sect. 4.2.2, it will be shown that the bimodal luminosity distribution is mostly a selection effect due to EO samples covering solely the high luminosity regime.

Figure 4 shows two histograms of the number of EOs with different effective radii. For EOs in both early- and late-type galaxies, the majority of objects has small effective radii. The mean size for EOs in early-type galaxies is $18.1 \mathrm{pc}$ and its median value lies at $14.2 \mathrm{pc}$. For EOs in early-type galaxies that are fainter than $M_{\mathrm{V}}=-10 \mathrm{mag}$, the mean and the median size are $15.2 \mathrm{pc}$ and $13.5 \mathrm{pc}$, respectively. The sizes of EOs in late-type galaxies are slightly smaller: the mean size is $14.4 \mathrm{pc}$ and its median value is $13.2 \mathrm{pc}$. For EOs in late-type galaxies, $39.2 \%$ have effective radii between 10 and 12 pc. For early-type galaxies,

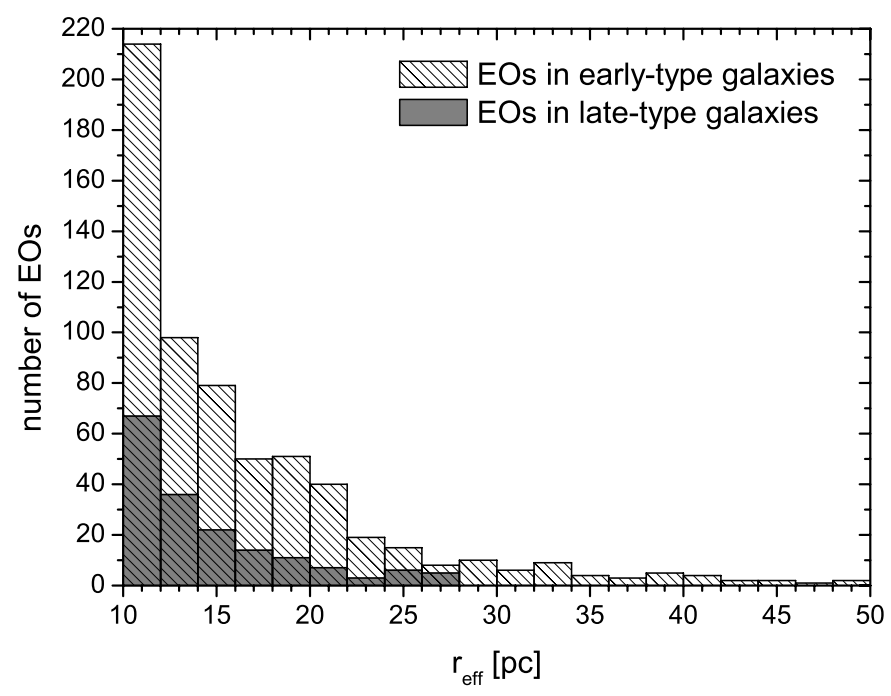

Fig. 4. Histogram of the number of EOs in early-type and late-type galaxies at different effective radii.

$38.8 \%$ and $25.0 \%$ are in the interval between 10 and $12 \mathrm{pc}$ for EOs fainter and brighter than $M_{\mathrm{V}}=-10 \mathrm{mag}$, respectively.

A histogram of the projected distance of EOs to their host galaxies is presented in Fig. 5. EOs in late-type galaxies were predominantly found at small projected distances below $20 \mathrm{kpc}$. It should be noted, however, that the coverage of the halo beyond projected distances of $20 \mathrm{kpc}$ is extremely poor for most latetype galaxies. EOs in early-type galaxies were found also at considerably larger distances. The mean and the median projected distances are $12.9 \mathrm{kpc}$ and $6.4 \mathrm{kpc}$ for EOs in late-type galaxies and $28.7 \mathrm{kpc}$ and $17.9 \mathrm{kpc}$ for EOs in early-type galaxies, respectively. Only few EOs have been discovered beyond $100 \mathrm{kpc}$. All EOs with projected distances larger than $130 \mathrm{kpc}$ are associated with the giant elliptical galaxies in the center of the Virgo Cluster, the Fornax Cluster, the Perseus Cluster, and the Coma Cluster. This result does not necessarily imply that only the central galaxies of clusters have EOs at very large distances, as 


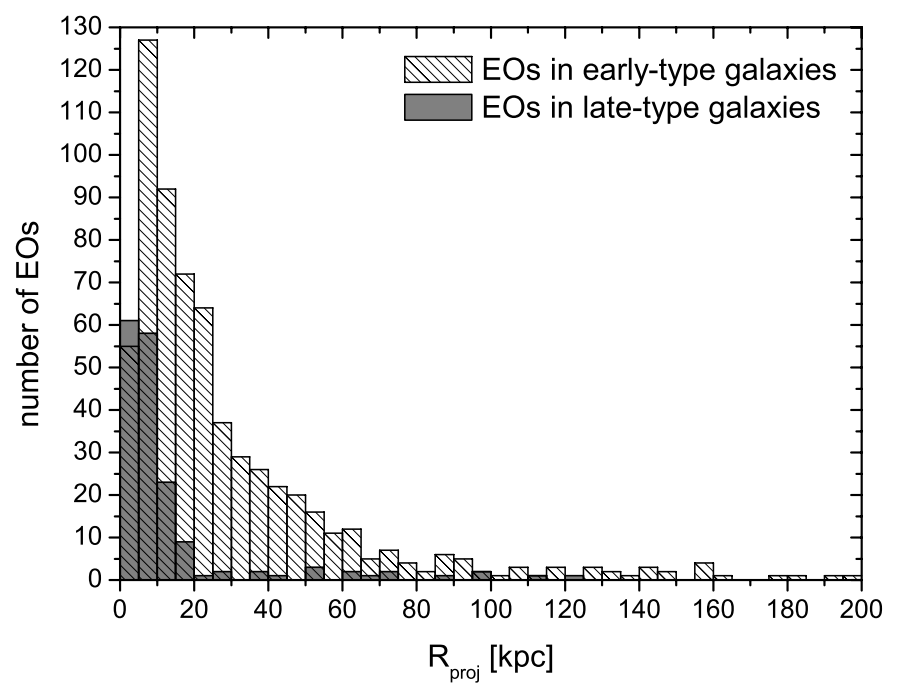

Fig. 5. Histogram of the number of EOs in early-type and late-type galaxies at different projected radii.

rather isolated galaxies scarcely have HST observations at such large projected distances (see Sect. 4.3).

\subsection{Correlation of EO parameters with those of their host galaxies.}

Figure 6 shows the dependence of the parameters projected distance of EOs to their host galaxies, $R_{\text {proj }}$, effective radius, $r_{\text {eff }}$, and absolute $V$-band magnitude of the EOs, $M_{\mathrm{V}, \mathrm{EO}}$, on the total $V$-band luminosity of the host galaxies, $M_{\mathrm{V}, \mathrm{gal}}$.

Figures $6 \mathrm{a}$ and $6 \mathrm{~b}$ illustrate the dependence of the projected distances of the EOs on the total $V$-band luminosity of the host galaxies. For EOs in late-type galaxies only three galaxies (the Local Group galaxies Milky Way, M 31, and M 33) host EOs at projected distances beyond $20 \mathrm{kpc}$. In contrast, for early-type galaxies with luminosities between -21 and -24 mag EOs are found at very large projected distances even beyond $100 \mathrm{kpc}$. Only very few objects are found at projected distances less than a few kiloparsecs. This lack of EOs at small projected distances is at least partly due to the fact that EOs have a very low contrast on the bright background light from the host galaxy.

Figures $6 \mathrm{c}$ and $6 \mathrm{~d}$ demonstrate that the upper limit of the effective radii of EOs in late-type and early-type galaxies tends to increase with the total $V$-band luminosity of the host galaxy. For all galaxies, most EOs have effective radii well below 20 pc.

Figure 6e shows that the total luminosity of EOs in latetype galaxies does not depend on the total luminosity of their host galaxy. For EOs in early-type galaxies (Fig. 6f) there is a trend: the more massive the parent galaxy the higher is the upper limit of the luminosities of its EOs. This trend is partly a size-ofsample effect, as in large EO samples also extreme luminosities can be realized. In Sect. 4.2.2 we will demonstrate, however, that the number of high-luminosity objects is larger than expected from the EO luminosity function.

Figure $7 \mathrm{a}$ plots the projected distances of the EOs versus the distance of their host galaxies from the Galaxy. Early-type galaxies have been studied nearby as well as at large distances up to $143 \mathrm{Mpc}$, while late-type galaxies were mainly observed in the local universe up to distances of $10 \mathrm{Mpc}$. Only one spiral (NGC 3370) was searched for EOs at a larger distance of about $27 \mathrm{Mpc}$.
Figure $7 \mathrm{~b}$ displays the absolute $V$-magnitude of the EOs versus the distance of the host galaxy from the Galaxy. The maximum absolute $V$-magnitude of EOs does not change with the distance of the host galaxy. While EOs brighter than $M_{\mathrm{V}}=$ -10 mag were detected at all distances, faint EOs have only been observed in galaxies closer than $30 \mathrm{Mpc}$.

\section{Discussion}

\subsection{Distribution in the $r_{\mathrm{eff}}$ vs. $M_{\mathrm{v}}$ space}

Figure 1 shows the effective radii $r_{\text {eff }}$ of the 813 EOs as a function of their total $V$-band luminosities $M_{\mathrm{V}}$. At all luminosities, EOs cover a range between $10 \mathrm{pc}$ and an upper limit, which increases with increasing luminosity from about $25 \mathrm{pc}$ at $M_{\mathrm{V}}=-5 \mathrm{mag}$ to about $100 \mathrm{pc}$ at $M_{\mathrm{V}}=-13 \mathrm{mag}$.

This trend of the increasing upper limit of effective radii with total luminosities defines the range where EOs have been found so far. It should not, however, be regarded as a firm upper limit, as very extended objects with a low total luminosity are extremely hard to detect. While the detection limit of EOs depends on numerous parameters like the magnitude and the structure of the fore- and background emission, we will focus on the characteristics of the EOs themselves.

The effective radius is defined as the radius where half of the total luminosity of an object is included. A large effective radius means that the luminosity is spread over a large area leading to low surface brightness. In order to provide a rough estimate of the mean surface brightness, we divide the luminosity within the effective radius by the area of a circle with the size of the effective radius,

$\overline{\Sigma_{\mathrm{V}, \mathrm{EO}}}=\frac{0.5 L_{\mathrm{V}, \mathrm{EO}}}{A\left(r_{\mathrm{eff}}\right)}=\frac{10^{-0.4\left(M_{\mathrm{V}}-M_{\mathrm{V}, \odot}\right)}}{2 \pi r_{\mathrm{eff}}^{2}}$

where $\overline{\Sigma_{\mathrm{V}, \mathrm{EO}}}$ is the mean surface brightness, $L_{\mathrm{V}, \mathrm{EO}}$ is the total luminosity of an EO, $M_{\mathrm{V}, \odot}=4.83$ mag is the absolute solar $V$-band luminosity, and $A\left(r_{\text {eff }}\right)=\pi r_{\text {eff }}^{2}$ is the area within $r_{\text {eff }}$. Figure 8 shows $r_{\mathrm{eff}}$ vs. $M_{\mathrm{V}}$ of EOs as in Fig. 1 and in addition lines with constant mean surface brightness as defined in Eq. (1). An EO with $r_{\text {eff }}=10 \mathrm{pc}$ and $M_{\mathrm{V}}=-5.0$ mag has the same mean surface brightness as an EO with $r_{\mathrm{eff}}=50 \mathrm{pc}$ and $M_{\mathrm{V}}=$ $-8.5 \mathrm{mag}$. On the other hand, Fig. 8 demonstrates that the trend of the increasing upper limits of effective radii with increasing total luminosity is not aligned with the lines of constant mean surface brightness. Consequently, the trend cannot be explained as a simple limit of detectability.

The EO catalog and the results presented in Sect. 3 contain both confirmed EOs and EO candidates. EOs are identified initially in HST images on the basis of an almost round shape and a color consistent with being a GC. The criteria exclude a fair fraction of background galaxies. However, the presence of round background galaxies with the same color as GCs cannot be excluded. Only in the very neighborhood of the Local Group, EOs can be resolved into stars discriminating them from background objects. Consequently, all more distant candidates need to be confirmed by follow-on spectroscopy measuring radial velocities of the objects. As such a procedure is extremely time consuming, only a fraction of the candidate GCs and EOs are observed spectroscopically.

The large extension of EOs leads to significantly lower central surface brightnesses. In terms of central surface brightness, an EO with $r_{\text {eff }}=10 \mathrm{pc}$ is roughly a factor of 25 or 3.5 mag fainter than a GC with the same total luminosity, 


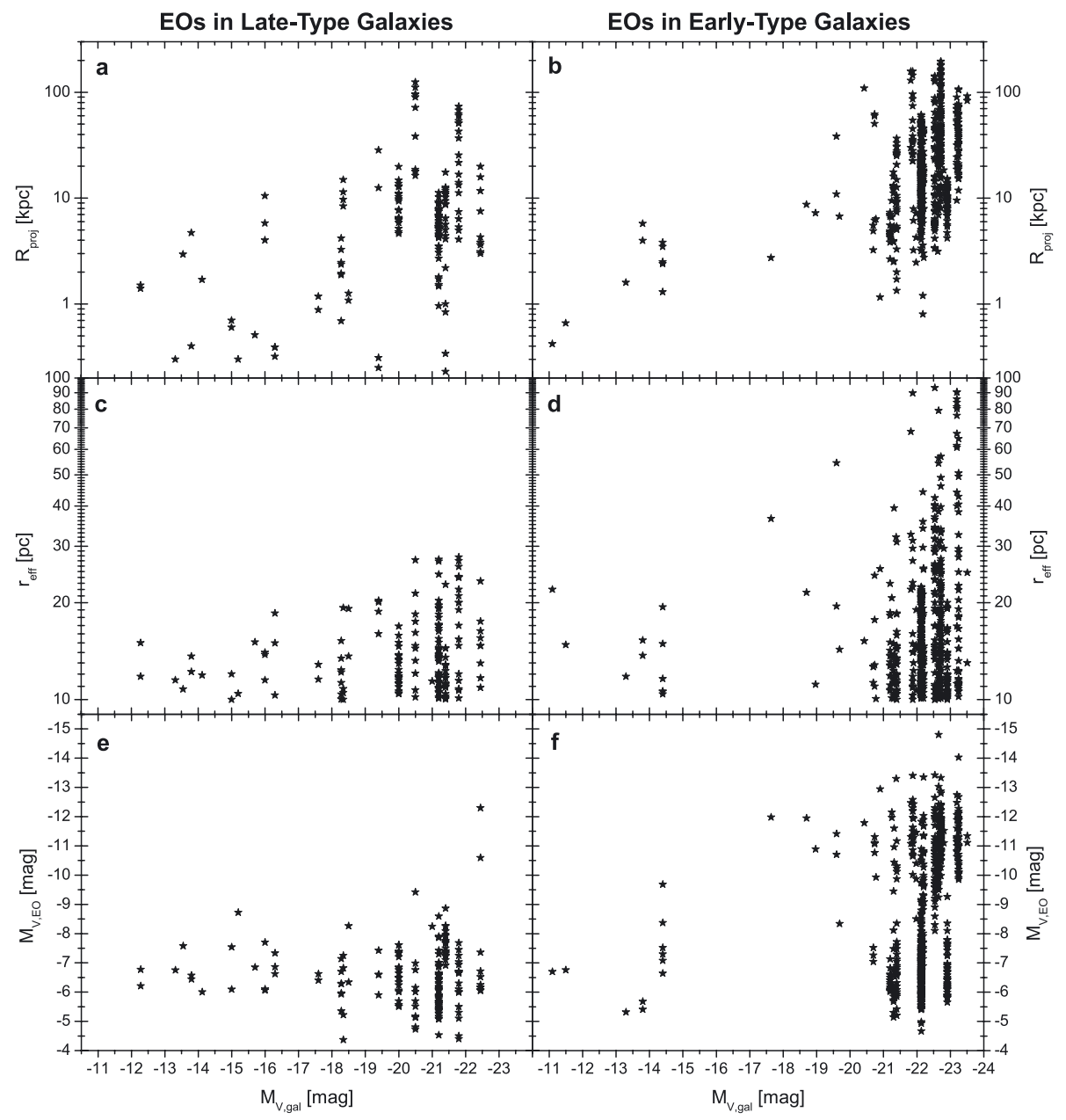

Fig. 6. a) and b) Projected distances of EOs are plotted against the total $V$-band luminosity of their host galaxies. c) and $\mathbf{d})$ Effective radii of EOs are plotted against the total $V$-band luminosity of the host galaxies. e) and f) Absolute $V$-magnitudes of EOs are plotted against the total $V$-band luminosity of the host galaxies. but with $r_{\text {eff }}=2$ pc. An EO with $r_{\text {eff }}=30 \mathrm{pc}$ is already a factor of about 225 or 5.9 magnitudes fainter. Consequently, GCs are typically preferred over EOs during the selection of targets for confirmation, as they are considerably easier to confirm.

From the 813 EOs in the catalog, only 175 EOs were so far spectroscopically confirmed. Figure 9 shows effective radii of confirmed (black) and candidate EOs (grey) as a function of their total $V$-magnitudes. The confirmed EOs cover basically the same $M_{\mathrm{V}}$ and $r_{\text {eff }}$ parameter space as the entire catalog and show the same trend of increasing upper size limits with increasing mass. Consequently, the overall distribution of EOs in the $r_{\text {eff }}$ vs. $M_{\mathrm{V}}$ space and the trend of increasing upper size-limits with increasing luminosity are not significantly influenced by contaminating background objects.

Figure 10 shows next to the effective radii and total luminosities of EOs also the corresponding parameters of GCs with effective radii smaller than $10 \mathrm{pc}$. The parameters of the GCs were taken from the same papers used for the EOs (see Sect. 2). The diagram demonstrates that EOs and GCs form a coherent structure in the $M_{\mathrm{V}}$ vs. $r_{\text {eff }}$ parameter space.

Figure 11 is a histogram of the effective radii of all star clusters presented in Fig. 10. The largest number of objects is contained in the bin covering effective radii between 2 and 4 pc. For larger effective radii, the number of objects decreases approximately exponentially. Star clusters with effective radii below 6 pc include 80 percent of all objects, while the EOs represent about 10 percent of the objects.

In Fig. 10, the median effective radii per luminosity bin of the combined GC-EO-sample are given as squares. For luminosities fainter than about $M_{\mathrm{V}}=-10.5$ mag, compact clusters with effective radii of a few parsec dominate. With increasing luminosity, EOs start to dominate over GCs leading to an overall trend of increasing effective radii with increasing total luminosity. The median effective radius increases from $10 \mathrm{pc}$ at $M_{\mathrm{V}}=-11.0 \mathrm{mag}$ to $30 \mathrm{pc}$ at $M_{\mathrm{V}}=-13.5 \mathrm{mag}$. At the high-luminosity end, the number of objects is quite low. The low median effective radius at $M_{\mathrm{V}}=-13.0 \mathrm{mag}$ is due to three very compact candidate GCs in this bin. The removal of these unconfirmed GCs results in a median effective radius that fits the overall trend (grey triangle). While the data show a clear trend of increasing effective radii with increasing luminosity, a tight size-luminosity relation as seen in older publications (e.g. Dabringhausen et al. 2008; Evstigneeva et al. 2008) is no longer existing on the basis of the larger data-set presented in this paper. This result is consistent with the conclusions of Brodie et al. (2011), which were based on a considerably smaller data-set.

Figure 10 shows next to the GCs and EOs (black circles) also the effective radii and absolute luminosities of early-type galaxies (open circles). We compiled the parameters of the elliptical, dwarf and compact elliptical, and dwarf spheroidal galaxies from Brasseur et al. (2011), Slater et al. (2011), Bell et al. (2011), McConnachie \& Irwin (2006), Cappellari et al. (2006), Sharina et al. (2008), Mieske et al. (2005), Huxor et al. (2011b), Belokurov et al. (2010), Misgeld \& Hilker (2011), Da Costa et al. (2009), Martin et al. (2008), Price et al. (2009), Geha et al. (2010), Smith Castelli et al. (2008), Smith Castelli et al. (2012), Blakeslee \& Barber (2008), and McConnachie (2012). The galaxies span a luminosity range of about 24 mag and a size range of three orders of magnitude. 
R. C. Brüns and P. Kroupa: A catalog of ECs and UCDs

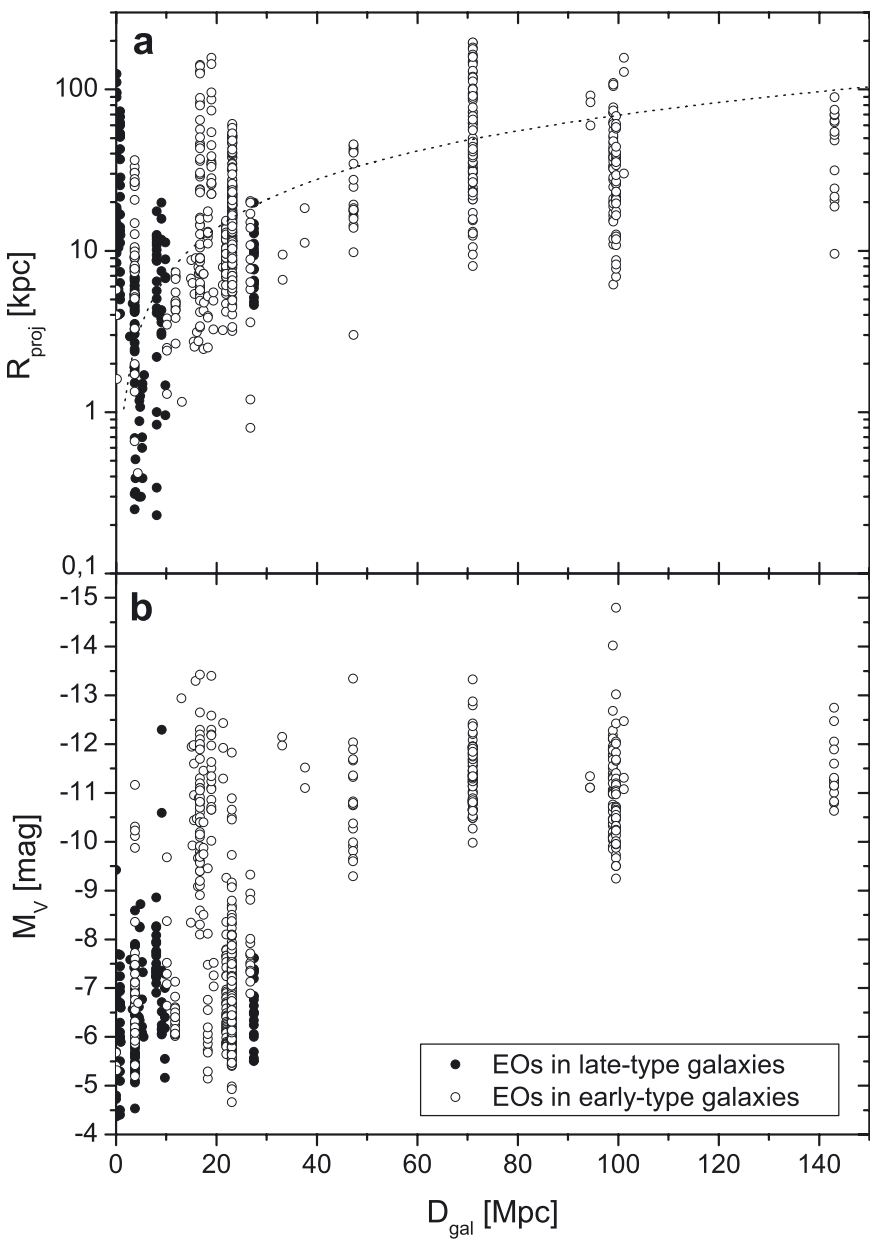

Fig. 7. a) Projected distances of EOs from their host galaxies are plotted against the distance of the host galaxies. The dotted curve indicates the largest possible projected radius within a single HST ACS image centered on a galaxy at a given distance. b) Absolute $V$-magnitudes of EOs are plotted against the distance of the host galaxies.

While the star clusters (GCs and EOs) and the early-type galaxies form both a coherent structure in the $M_{\mathrm{V}}$ vs. $r_{\mathrm{eff}}$ parameter space, there is a clear gap between star clusters and galaxies at least in the luminosity interval between $M_{\mathrm{V}}=-6 \mathrm{mag}$ and -11 mag. This gap was first discussed by Gilmore et al. (2007). At $M_{\mathrm{V}}=-6 \mathrm{mag}$, EOs have effective radii up to about $30 \mathrm{pc}$, while the dwarf spheroidal galaxies at this luminosity have effective radii between about 100 and $400 \mathrm{pc}$. In the high-luminosity region at about $M_{\mathrm{V}}=-12$ mag EOs have effective radii up to about $90 \mathrm{pc}$, while the dwarf galaxies at this luminosity have effective radii Between 200 and 1300 pc. between $M_{\mathrm{V}}=-11$ and $-12 \mathrm{mag}$ there are six candidate compact ellipticals from Blakeslee \& Barber (2008). They have the same parameters as EOs, but they are slightly larger than $100 \mathrm{pc}$, which lead to the classification as cEs. As there is no clear distinction between EOs and cEs, these six cEs might as well be very extended EOs.

The ultra faint dwarf galaxies (UFDs), which were recently found around the Milky Way (e.g. Martin et al. 2008; Belokurov et al. 2010), cover the luminosity range between $M_{\mathrm{V}}=0$ and $-5 \mathrm{mag}$. The detection of large objects with effective radii greater than $20 \mathrm{pc}$ and luminosities fainter than $M_{\mathrm{V}}=-5$ mag is extremely challenging even within the Local Group. While there is no overlap between UFDs and EOs for the Milky Way, a potential overlap of EOs and UFDs for other galaxies cannot be

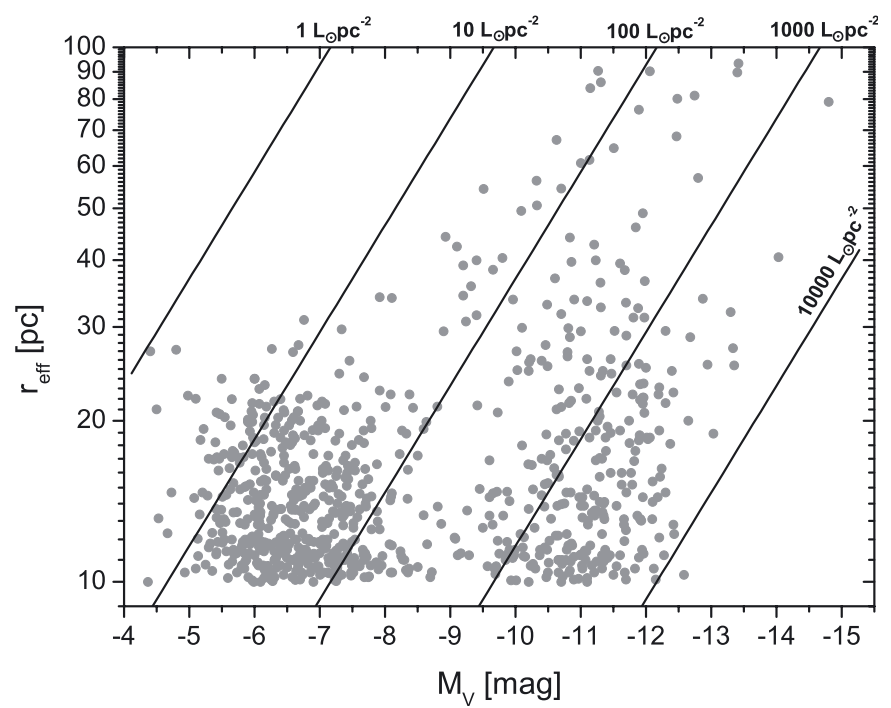

Fig. 8. Effective radii of EOs are plotted against their absolute $V$-magnitudes (grey circles). The black lines indicate trends of equal mean surface brightnesses of $1,10,100,1000$, and $10000 L_{\odot} \mathrm{pc}^{-2}$.

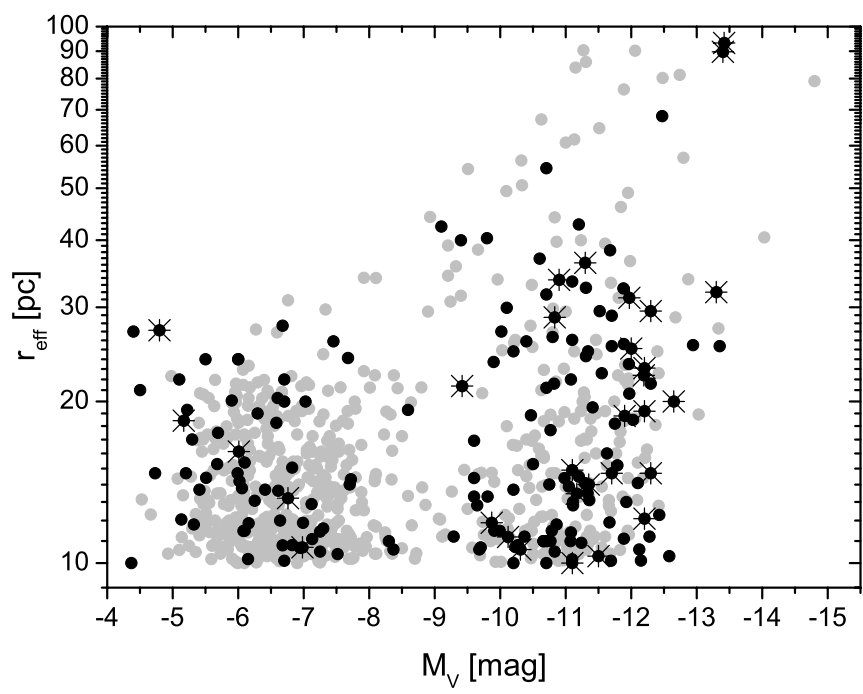

Fig. 9. Effective radii of EOs are plotted against their absolute $V$-magnitudes. Black circles indicate the 175 confirmed EOs, while grey circles are the $638 \mathrm{EO}$ candidates. The $31 \mathrm{EOs}$ which have a measured dynamical mass are marked by an additional star.

excluded, as neither UFDs nor very faint EOs were within the detection limits of existing surveys.

On the high-luminosity end of the EO distribution, some compact elliptical galaxies have parameters comparable to the most extended EOs. Figure 10 shows two EOs brighter than $M_{\mathrm{V}}=-14 \mathrm{mag}$, with effective radii of about 40 and $80 \mathrm{pc}$ (Madrid et al. 2010; Madrid 2011). However, both are so far unconfirmed candidates that might be background galaxies. The three most luminous, confirmed objects having $M_{\mathrm{V}} \approx-13.4 \mathrm{mag}$ are VUCD7, UCD3, and HUCD1, which are associated with the central elliptical galaxies of the Virgo Cluster, M 87, of the Fornax Cluster, NGC 1399, and the Hydra Cluster, NGC 3311, respectively. In addition, the confirmed object $\mathrm{M} 59 \mathrm{cO}$, which is associated with the giant elliptical galaxy M59, has a luminosity of about $M_{\mathrm{V}} \approx-13.3 \mathrm{mag}$. VUCD7 and UCD3 have effective radii on the order of $90 \mathrm{pc}$, while HUCD1 and M59cO have effective radii of 25 and $32 \mathrm{pc}$, respectively. 
A\&A 547, A65 (2012)

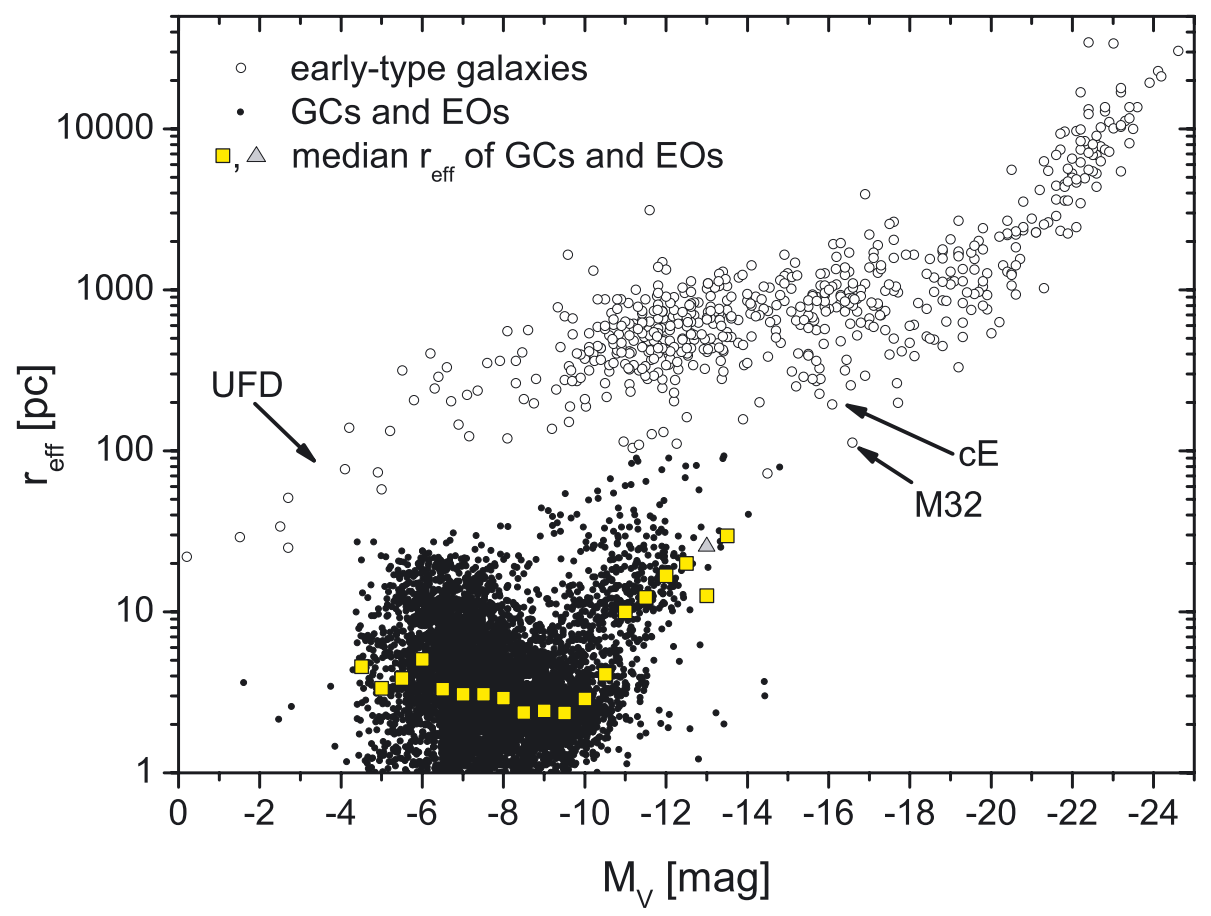

Fig. 10. Effective radii of GCs and EOs are plotted against their absolute $V$-magnitudes (black circles). The median effective radius per luminosity bin is given as squares. The six exceptionally bright GCs with luminosities between $M_{\mathrm{V}}=-12.5$ and $-14.5 \mathrm{mag}$ and effective radii below $4 \mathrm{pc}$ are unconfirmed candidate clusters associated with NGC 4365 (Blom et al. 2012). The grey triangle shows the median effective radius, when these exceptionally bright compact GCs were removed in the bin at $M_{\mathrm{V}}=-13.0 \mathrm{mag}$. In addition, early-type galaxies are shown as open circles. Highlighted are the two galaxy types compact ellipticals (cE) with their prototype example M 32, and the ultra faint dwarf galaxies (UFD) recently found in the Milky Way.

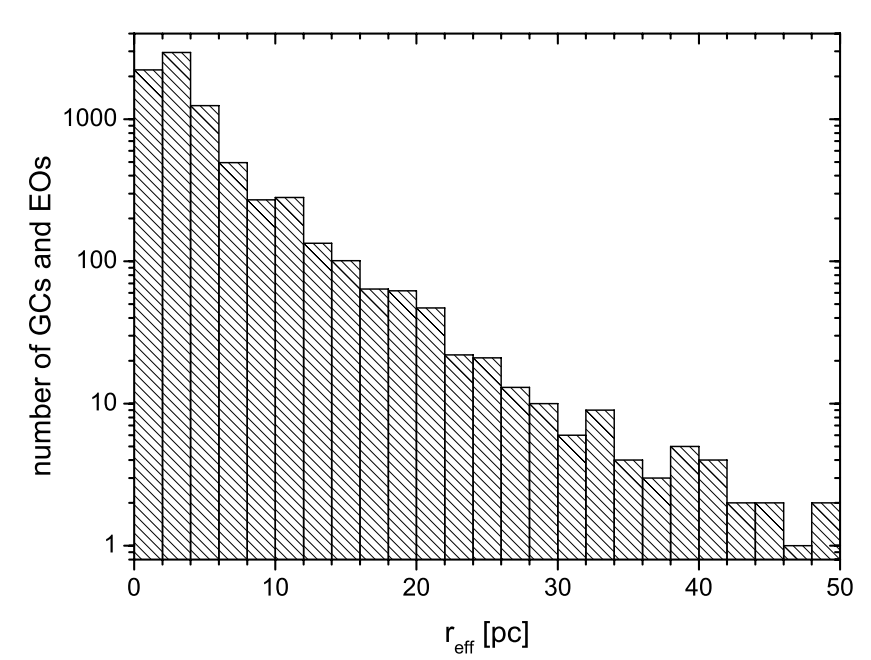

Fig. 11. Histogram of the number of star clusters at different effective radii for all GCs and EOs shown in Fig. 10. The slight increase of numbers at $10 \mathrm{pc}$ is due to the fact that all publications of this paper were selected to contain EOs, but not all of them also include GCs.

The Coma Cluster compact elliptical galaxy CcGV19b (Price et al. 2009), which has a luminosity of $M_{\mathrm{V}} \approx-14.5 \mathrm{mag}$ and an effective radius of $r_{\mathrm{eff}}=72 \mathrm{pc}$, is located at a projected distance of $68 \mathrm{kpc}$ to NGC 4874. These parameters could also lead to a classification of CcGV19b as an EO. The observed mass-to-light ratio of about 13 (Price et al. 2009), which is about a factor of three larger than that of VUCD7 and UCD3 (Evstigneeva et al. 2007; Mieske et al. 2008), suggests rather a galactic origin. However, the vast majority of EOs, about $96 \%$, do not have observed velocity dispersions (see Fig. 9), which are needed to estimate a dynamical mass. Consequently, some overlap between EOs and compact elliptical galaxies cannot be excluded.

\subsection{EO luminosity functions}

A common way of parameterization of samples of astronomical objects are luminosity functions. Secker (1992) analyzed compact GCs in the Milky Way and M31 and concluded that their luminosity functions are well represented by so-called Student $t_{5}$ functions

$L F \propto\left[1+\frac{\left(M_{\mathrm{V}}-M_{\mathrm{V}, \mathrm{TO}}\right)^{2}}{5 \sigma_{\mathrm{V}}^{2}}\right]^{-3}$,

where $M_{\mathrm{V}}$ are absolute $V$-band magnitudes of star clusters, $M_{\mathrm{V}, \mathrm{TO}}$ is the turnover of the luminosity function and $\sigma_{\mathrm{V}}$ is the dispersion of the $t_{5}$ function. For compact GCs, the turnover of the luminosity function, $M_{\mathrm{V}, \mathrm{TO}}$, is very constant for various types of galaxies, making it a reasonable distance estimator (see Rejkuba 2012, and references therein). The mean turnover luminosity for the Milky Way and 18 nearby galaxies is $M_{\mathrm{V}, \mathrm{TO}}=$ $-7.66 \pm 0.09 \mathrm{mag}$.

\subsubsection{EOs in late-type galaxies}

A histogram of the number of EOs at different total luminosities of late-type galaxies is shown in Fig. 12. A fit of the luminosity function according to Eq. (2) is added. The turnover of the luminosity function is $M_{\mathrm{V}, \mathrm{TO}}=-6.47 \pm 0.03 \mathrm{mag}$ and the dispersion of the $t_{5}$ function is $\sigma_{\mathrm{V}}=0.91 \pm 0.03 \mathrm{mag}$. The peak of the EO luminosity function is about one magnitude fainter than the typical peak of the GC luminosity function.

For the histogram in Fig. 12 all 171 EOs associated with late-type galaxies are used. As discussed in Sect. 4.1, only a fraction of EOs were confirmed by follow-on spectroscopy. Consequently, it cannot be excluded that some background galaxies modified the exact result of the luminosity function. The number of confirmed EOs in late-type galaxies is 43 . The mean total luminosity of the 43 EOs is $M_{\mathrm{V}}=-6.40$ mag. Considering the low number of objects, this value is quite close to the fitted value for all candidate EOs, indicating that the EOs in late-type galaxies indeed have a fainter peak of the luminosity function than compact GCs.

Considering the very low surface brightness of faint and extended EOs (see Sect. 4.1), a fair fraction of very extended and faint EOs is most likely below the detection limit of 


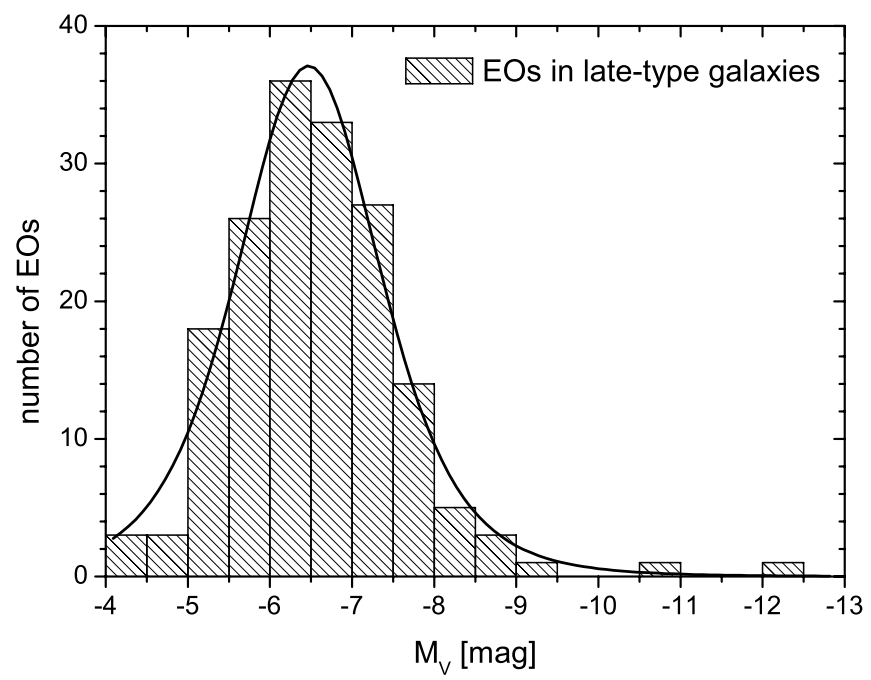

Fig. 12. Histogram of the number of EOs in late-type galaxies at different total $V$-magnitudes. The black line represents a fitted $t_{5}$ luminosity function.

extra-galactic surveys. The true turnover of the EO luminosity function might therefore be at even lower luminosities.

\subsubsection{EOs in early-type galaxies}

Figure 3 shows that EOs in early-type galaxies show a bimodal distribution which peaks at about $M_{\mathrm{V}}=-6.5 \mathrm{mag}$ and $-11.0 \mathrm{mag}$ and has a clear minimum between -8.5 and -9 mag. On the other hand, Fig. $7 \mathrm{~b}$ demonstrates that for a large fraction of early-type galaxies only high-luminosity objects were considered. This is partly due to detection limits especially at large distances, but also due to the fact that since the discovery of UCDs in the Fornax Cluster by Hilker et al. (1999) and Drinkwater et al. (2000), much effort has been made to detect and to analyze EOs brighter than about $M_{\mathrm{V}}=-10 \mathrm{mag}$, while fainter EOs were neither in the focus of UCD studies nor in those investigating GCs.

A number of GC surveys applied size limits to reduce the contamination of background galaxies. For instance, the GC surveys covering 100 galaxies of the Virgo Cluster (Jordán et al. 2005) and 43 galaxies of the Fornax Cluster (Masters et al. 2010) applied a size limit of $r_{\mathrm{eff}}<10 \mathrm{pc}$ to reduce the contamination by background galaxies. As a side effect, they excluded also all EOs from their GC catalogs.

The very different approaches for objects with low and high luminosities have a significant influence on the luminosity function. One example is the galaxy M 85 of the Virgo Cluster. Four EOs are found with luminosities brighter than $M_{\mathrm{V}}=-8.5 \mathrm{mag}$ (Haşegan et al. 2005; Chies-Santos et al. 2011). Peng et al. (2006) used the same Virgo Cluster survey data as Jordán et al. (2005) to search for diffuse star clusters and concluded that the galaxy M 85 has about 30 EOs with luminosities between $M_{\mathrm{V}}=$ -5.5 and $-8.5 \mathrm{mag}$. While the low luminosity objects would dominate for this galaxy, our catalog contains only the four bright M 85 EOs of Haşegan et al. (2005) and Chies-Santos et al. (2011) as Peng et al. (2006) have not published a catalog of their EOs. In the same field of view Jordán et al. (2005) found 211 compact GCs in M 85. For this specific galaxy, the EOs would add about $15 \%$ to the GC sample.

Another example is the giant elliptical galaxy NGC 4365, which was covered by eight HST ACS fields (Blom et al. 2012). Seven HST ACS fields provide a very good coverage

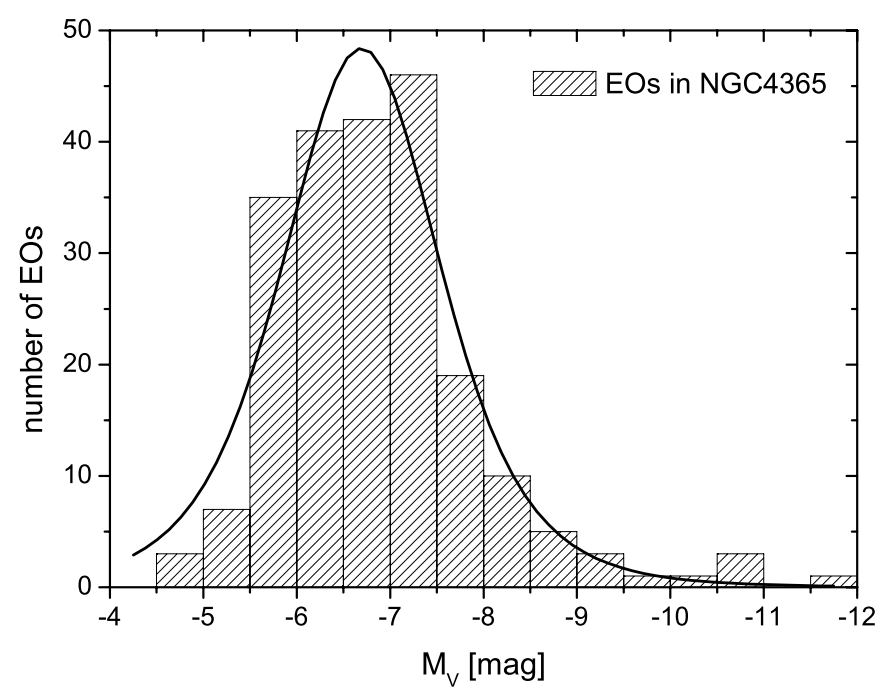

Fig. 13. Histogram of the number of EOs in the elliptical galaxy NGC 4365 at different total $V$-magnitudes. The black line represents a fitted $t_{5}$ luminosity function.

of the inner $35 \mathrm{kpc}$ of the galaxy while the last field pointed to the halo delivering clusters with projected distances between about 40 and $60 \mathrm{kpc}$. Blom et al. (2012) found in total $2038 \mathrm{GC}$ and 217 EO candidates. For this galaxy, EOs add about 10 percent to the GC sample. Only 5 of the 217 EOs are brighter than $M_{\mathrm{V}}=-10 \mathrm{mag}, 19$ EOs have a luminosity between $M_{\mathrm{V}}=$ -8 and $-10 \mathrm{mag}$, and 148 EOs have one between $M_{\mathrm{V}}=-6$ and -8 mag. Again, the number of low-luminosity EOs is significantly larger than the number of bright EOs. The EO candidates of NGC 4365 form the largest EO sample of an elliptical galaxy. Figure 13 shows the histogram of the number of EO candidates of NGC 4365 as a function of luminosity. The black line shows the corresponding luminosity function having a turnover luminosity of $M_{\mathrm{V}, \mathrm{TO}}=-6.69 \pm 0.07$ and a dispersion of the $t_{5}$ function of $\sigma_{\mathrm{V}}=0.88 \pm 0.07 \mathrm{mag}$. The turnover luminosity is at slightly brighter luminosities as for the late-type galaxies.

In their central HST field, Blom et al. (2012) found $681 \mathrm{com}-$ pact clusters and $30 \mathrm{EOs}$, i.e. the EOs add about $4.4 \%$ to the GC sample in the central region of NGC 4365. For the 100 Virgo galaxies Jordán et al. (2005) found in single HST fields centered on the individual galaxies in total 12763 compact GCs. An EO fraction of 5\% would yield for these 100 galaxies an EO population of 638 EOs, which is about ten times the number of high luminosity objects in our catalog associated with these Virgo Cluster galaxies.

Consequently, an interpretation of the bimodal luminosity distribution needs to take into account the varying number of galaxies building the sample at each luminosity bin. Only seven early-type galaxies have observations of EOs fainter than $M_{\mathrm{V}}=$ -8.5 mag and only four galaxies have observations of EOs fainter than $M_{\mathrm{V}}=-6.5$ mag. For EO luminosities from $M_{\mathrm{V}}=$ $-8.5 \mathrm{mag}$ to $-10.5 \mathrm{mag}$, the number of observed galaxies increases from 9 to 20 . EOs brighter than $M_{\mathrm{V}}=-10.5$ mag were observed in 33 galaxies.

Figure 14 takes the varying number of galaxies into account, i.e. the number of EOs in each bin is divided by the number of galaxies contributing to this luminosity bin. The second peak at high luminosities has decreased considerably, but it has not entirely vanished, demonstrating that the large number of high-luminosity EOs associated with the most luminous galaxies is not a simple size-of-sample effect. A fit of the luminosity 


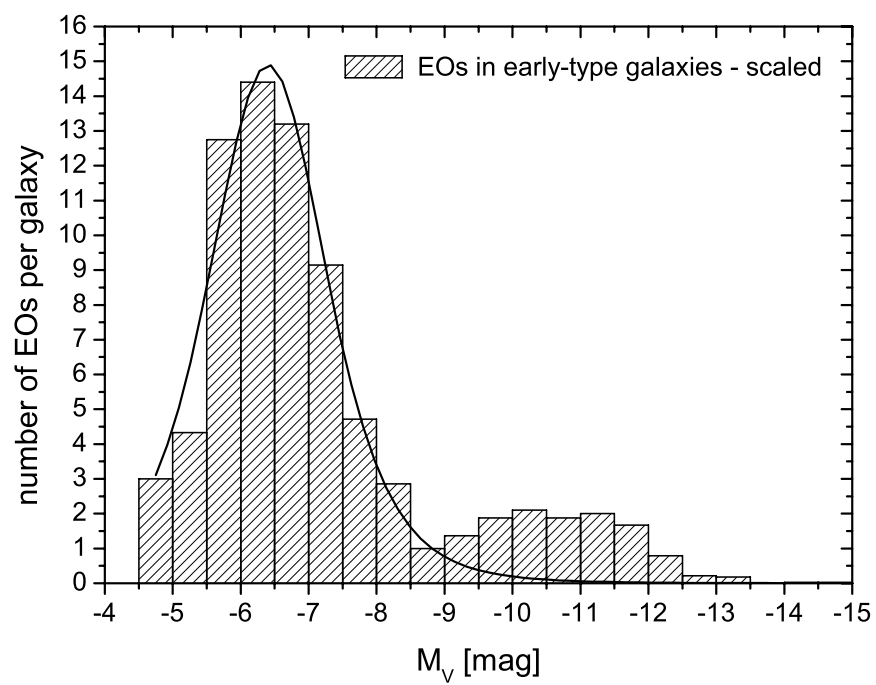

Fig. 14. Histogram of the normalized number of EOs in early-type galaxies at different total $V$-magnitudes scaled by the number of galaxies having observations at the individual bins. The black line represents a fitted $t_{5}$ luminosity function.

function according to Eq. (2) is added to Fig. 14. The peak of the luminosity function is at $M_{\mathrm{V}, \mathrm{TO}}=-6.40 \pm 0.06 \mathrm{mag}$ and the dispersion of the $t_{5}$ function is $\sigma_{\mathrm{V}}=0.89 \pm 0.07 \mathrm{mag}$. The values change only slightly to $M_{\mathrm{V}, \mathrm{TO}}=-6.40 \pm 0.05 \mathrm{mag}$ and $\sigma_{\mathrm{V}}=0.87 \pm 0.06 \mathrm{mag}$ when the high-luminosity tail (i.e. objects brighter than $M_{\mathrm{V}}=-9$ mag) is excluded from the fit.

The EO sample of early-type galaxies is dominated by the two galaxies NGC 4365 and NGC 1275, which have 217 and 84 EO candidates, respectively. In order to verify that the results for early-type galaxies are not biased toward these two galaxies, we have repeated the exercise of scaling the number of objects per luminosity bin by the number of relevant galaxies excluding NGC 4365 and NGC 1275 from the sample. Figure 15 shows the resulting histogram and a fitted luminosity function having $M_{\mathrm{V}, \mathrm{TO}}=-6.32 \pm 0.10 \mathrm{mag}$ and $\sigma_{\mathrm{V}}=0.89 \pm 0.12 \mathrm{mag}$. The results of the sample without NGC 4365 and NGC 1275 agree well with the results of the entire EO sample of early-type galaxies.

The results for early-type galaxies are very similar to the results from late-type galaxies. The main difference between the luminosity functions is the tail of high-luminosity objects associated with early-type galaxies.

Mieske et al. (2012) studied a sample of confirmed GCs and UCDs to adress the question whether there is an over-population of UCDs with respect to compact GCs. They concluded that the number of UCDs is consistent with a continuation of the GC luminosity function toward bright magnitudes. In this paper, we demonstrate that there is an over-population of bright EOs if compared with the EO luminosity function. While the Mieske et al. (2012) sample is well defined in the sense that all objects were spectroscopically confirmed, most of them have no measured size. They used all UCDs irrespective of their size and compared the number of UCDs with the GC luminosity function which has a turnover luminosity that is about one magnitude brighter than the EO luminosity function. In additon, the number of GCs is about 10 times larger than the number of EOs. Consequently, the results of Mieske et al. (2012) cannot easily be compared with our results as the samples are largely independend from each other. Larger EO and GC samples of a number of early type galaxies covering the entire luminosity range

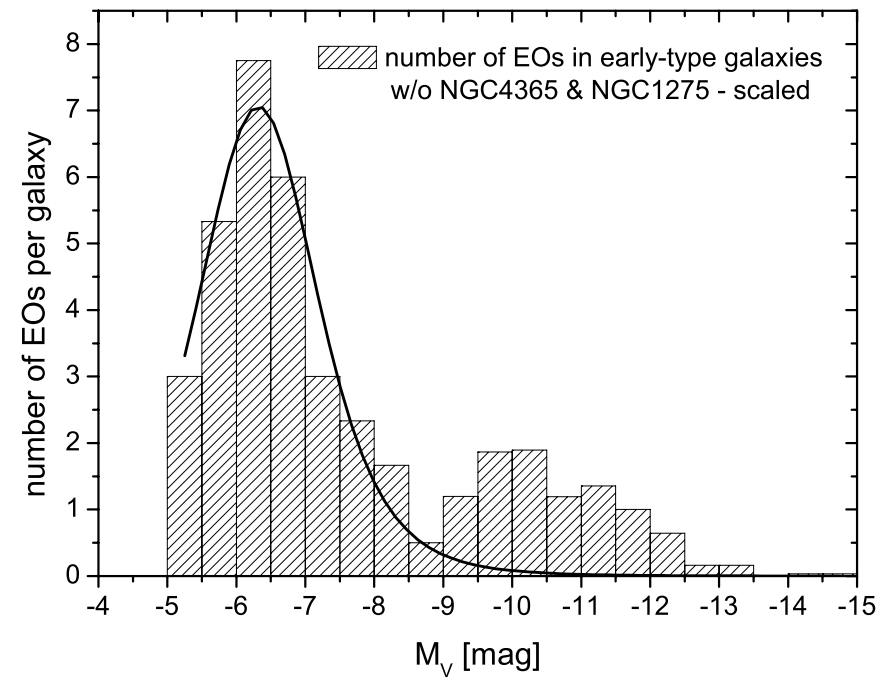

Fig. 15. Histogram of the normalized number of EOs in early-type galaxies except for the large EO samples of NGC 4365 and NGC 1275 at different total $V$-magnitudes scaled by the number of galaxies having observations at the individual bins. The black line represents a fitted $t_{5}$ luminosity function.

from $M_{\mathrm{V}}=-4$ to -14 mag are needed to answer the question whether there is a general overpopulation of bright EOs in earlytype galaxies or whether it is a specific feature seen only in special environments.

\subsubsection{Trends of the turnover luminosity with effective radius}

In the previous sections, we concluded that EOs in early and late-type galaxies have basically the same turnover of the luminosity function, which is about one magnitude fainter than that of compact GCs.

In this section, we explore the trend of the turnover luminosity with increasing effective radii. In order to increase the number of objects per $r_{\text {eff }}$-bin, we combine the data for GCs and EOs of early and late-type galaxies. We exclude the brightest objects in the UCD regime to avoid the second peak as seen in Figs. 3 and 14 and focus on objects fainter than $M_{\mathrm{V}}=-9$ mag.

For the compact GCs with effective radii below $6 \mathrm{pc}$, we derive a turnover of the luminosity function of $M_{\mathrm{V}, \mathrm{TO}}=-7.67 \pm$ $0.11 \mathrm{mag}$, which is in very good agreement with the mean turnover luminosity for the Milky Way and 18 nearby galaxies, $M_{\mathrm{V}, \mathrm{TO}}=-7.66 \pm 0.09 \mathrm{mag}$ (Rejkuba 2012).

Figure 16 shows the trend of the turnover luminosity as a function of the effective radius. The turnover luminosity decreases continuously from $M_{\mathrm{V}, \mathrm{TO}}=-7.67 \mathrm{mag}$ to $M_{\mathrm{V}, \mathrm{TO}}=$ -6.66 mag at the $r_{\text {eff }}$-bin between 10 and 12 pc. For larger effective radii, the turnover luminosity decreases considerably slower to values of $M_{\mathrm{V}, \mathrm{TO}}=-6.40 \mathrm{mag}$ at the $r_{\mathrm{eff}}$-bin between 20 and $30 \mathrm{pc}$.

On the basis of the available data, we conclude that the turnover of the luminosity function depends significantly on the effective radii of star clusters and that the slope of the varying $M_{\mathrm{V}, \mathrm{TO}}$ is steeper for GCs than for EOs.

However, the edge in the luminosity function at $M_{\mathrm{V}}=$ $-5.0 \mathrm{mag}$ for late-type and at $M_{\mathrm{V}}=-5.5 \mathrm{mag}$ for earlytype galaxies indicates that the samples are fairly incomplete at very low luminosities. The true turnover of the EO luminosity function is therefore expected to be at even lower luminosities, which might lead to a steeper slope in the EO regime. 


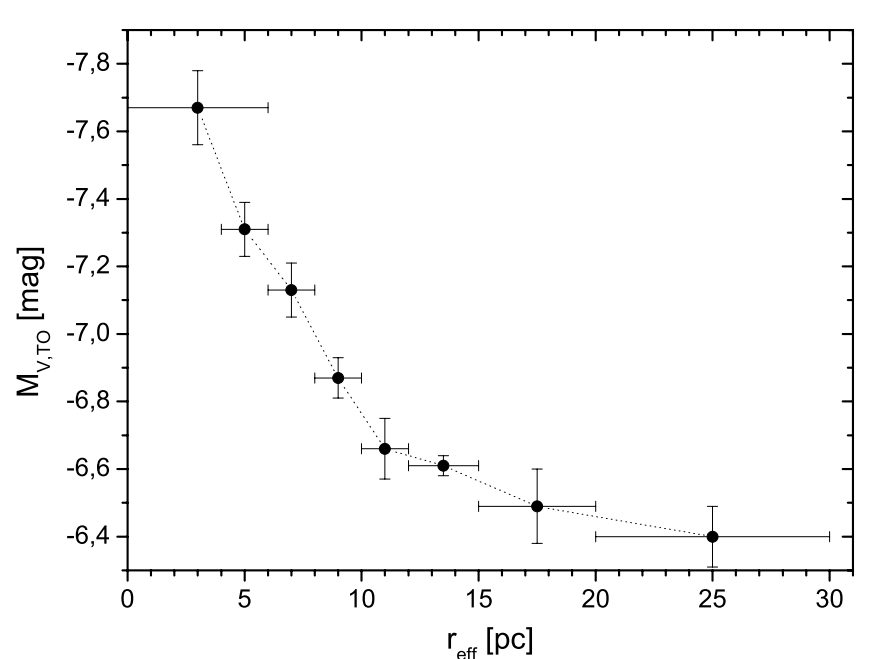

Fig. 16. Turnover of the luminosity function, $M_{\mathrm{V}, \mathrm{TO}}$, of GCs and EOs plotted as a function of the effective radius. The error bars in $r_{\text {eff }}$ indicate the individual bin-sizes, the error bars in $M_{\mathrm{V}, \mathrm{TO}}$ show the statistical error of the fit of the luminosity function to the data.

Considerably larger and more complete data-sets especially at low-luminosities, consisting of confirmed star clusters, are necessary to confirm the trend of the luminosity functions from GCs to EOs as shown in Fig. 16 and to substantiate the idea that this trend is a general feature of GCs and EOs in early- and late-type galaxies.

\subsection{Spatial distribution of EOs}

In the Milky Way, the 11 EOs have galacto-centric distances between 16 and $125 \mathrm{kpc}$ with a median distance of $72 \mathrm{kpc}$. In contrast, the GCs of the Milky Way are at considerably lower distances. The median distance of the GCs is $4.8 \mathrm{kpc}$. For M 31 five out of 20 EOs have projected distances smaller than $10 \mathrm{kpc}$ and 11 EOs have projected distances larger than $20 \mathrm{kpc}$ (Peacock et al. 2009; Huxor et al. 2008). The two EOs associated with M 33 have projected distances of 12.5 and $28.4 \mathrm{kpc}$ (Stonkute et al. 2008; Huxor et al. 2009).

The most serious constraint of EO catalogs of galaxies outside the Local Group is the very limited field of view of the HST ACS instrument, as EOs at large distances from the Galaxy can only be spatially resolved by HST. The field of view of the ACS instrument is $202^{\prime \prime}$ and the pixel size is $0.05^{\prime \prime}$.

The dotted curve in Fig. 7a indicates the largest possible projected radius of a single HST ACS image centered on a galaxy at a given distance. It visualizes the area covered by one Hubble field. At small distances a couple of HST ACS fields are necessary to scan a galaxy for EOs, whereas at large distances the galaxy and part of the halo are completely covered by one HST ACS field.

At the distance of the Whirlpool Galaxy M51 of about $8 \mathrm{Mpc}$, the field of view of $202^{\prime \prime}$ and the pixel size of $0.05^{\prime \prime}$ correspond to $7.8 \mathrm{kpc}$ and $2 \mathrm{pc}$, respectively. While the pixel size is well suited to resolve EOs, a number of ACS images are needed to cover the entire galaxy. Figure 17 shows an image of the Digitized Sky Survey $2\left(\mathrm{DSS}^{1}\right)^{1}$ of M 51 and the area covered by the mosaic of six ACS images used by Hwang \& Lee (2008) to find EOs in M 51, demonstrating that even six HST

\footnotetext{
1 The Digitized Sky Survey data used in this paper have been taken from the ESO Archive, see http://archive.eso.org/dss/dss
}

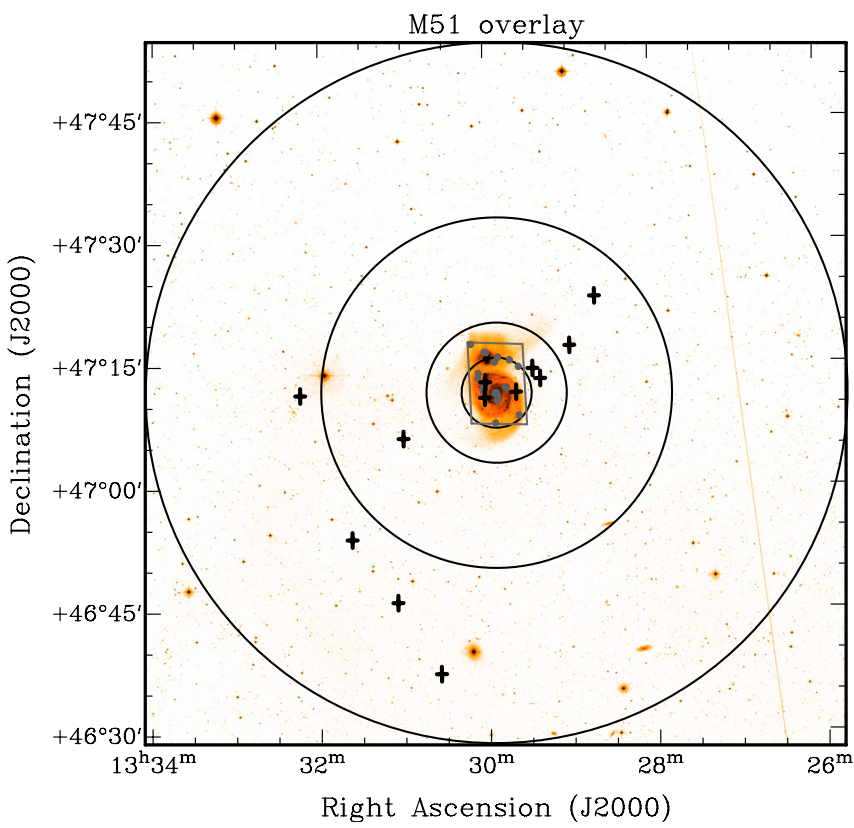

Fig. 17. M 51 EOs (grey dots) overlayed on a Digitized Sky Survey 2 (DSS2) image of M51. The grey lines indicate the size of the HST mosaic of 6 ACS images used by Hwang \& Lee (2008) to find EOs in M 51. The black circles indicate projected distances from M 51 of $10,20,50$, and $100 \mathrm{kpc}$. For comparison, the projected position of the Milky Way EOs, if the Milky Way would be seen face-on at the distance and the position of M 51, are added as black crosses.

ACS images do not cover the entire stellar body of the interacting galaxy pair.

The HST mosaic of M51 covers an area of $15.7 \mathrm{kpc} \times 23.6 \mathrm{kpc}$. This coverage is well suited to detect EOs related to the stellar bodies of the two galaxies and EOs in the lower halo. While halo EOs at considerably larger distances from their host galaxies might be found by chance in projection to the main body of the host galaxy, the probability is relatively low. Considering a line-of-sight of $\pm 100 \mathrm{kpc}$, the volume covered by the HST mosaic is $15.7 \times 23.6 \times$ $200 \mathrm{kpc}^{3}$, or $7.4 \times 10^{4} \mathrm{kpc}^{3}$. In contrast, the volume with a radius of $100 \mathrm{kpc}$, which would enclose the EOs, is $\frac{4}{3} \pi 100^{3} \mathrm{kpc}^{3}$ or $4.2 \times 10^{6} \mathrm{kpc}^{3}$. The HST mosaic of six ACS images covers less than 2 percent of the volume expected to contain EOs.

Figure 17 shows for comparison also the location of the Milky Way EOs, if the Milky Way would be seen face-on at the distance and the position of M 51. The six most distant EOs of the Milky Way have large galacto-centric distances between 70 and $125 \mathrm{kpc}$, or projected distances between 41 and $84 \mathrm{kpc}$ in Fig. 17. This figure demonstrates that only 3 of 11 EOs would be located within the HST mosaic. Consequently, also a number of EOs of M51 are expected to have considerably larger projected distances beyond the currently covered survey area.

In addition to EOs located in galactic halos, Larsen \& Brodie (2000) and Brodie \& Larsen (2002) have discovered a population of EOs co-rotating with the disk of the lenticular galaxy NGC 1023. These so-called faint fuzzies have similar structural parameters as halo EOs and are therefore not easily distinguishable from halo EOs projected onto the disk on the basis of imaging data alone. A fair fraction of EOs found in extragalactic surveys - especially those covering only the disk regions like the M 51 survey (Hwang \& Lee 2008) - might therefore be associated with the disks and not the halos of these galaxies. 


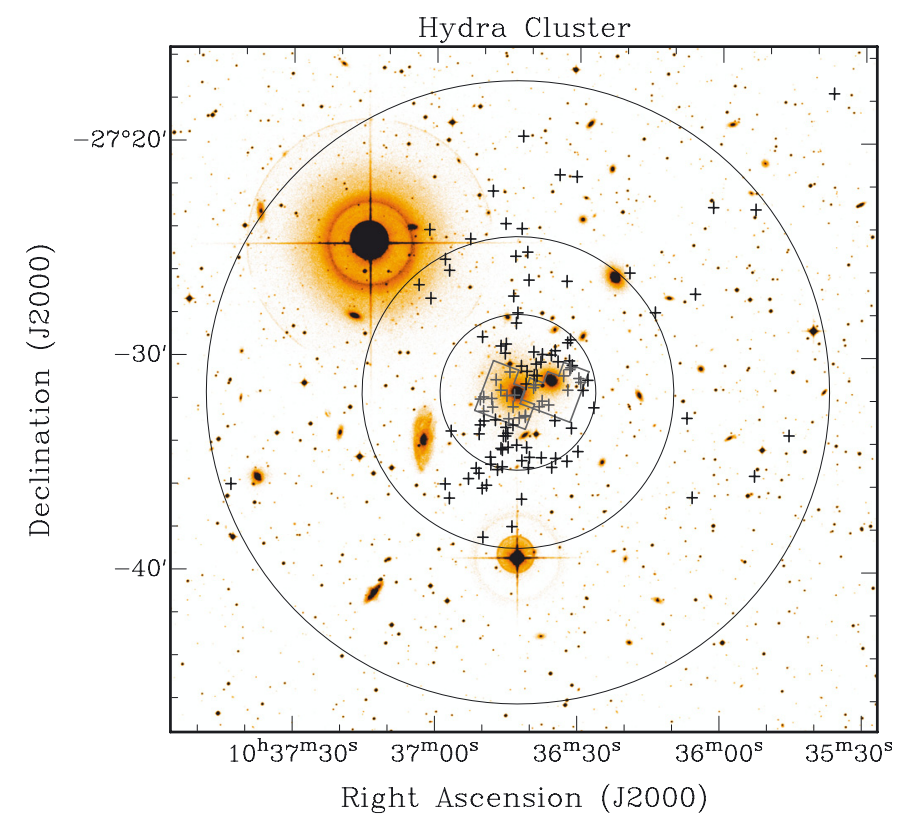

Fig. 18. Spectroscopically confirmed star clusters (crosses) of the Hydra Cluster (Misgeld et al. 2011) overlaid on a DSS2 image of the Hydra Cluster. Only those clusters which are located within the two HST WFPC 2 fields (marked by grey lines) have measured sizes. The black circles indicate projected distances from the central galaxy NGC 3311 of 50,100 , and $200 \mathrm{kpc}$.

The Hydra Cluster is located at a distance of about 47.2 Mpc. Misgeld et al. (2011) searched with ground based telescopes for massive star clusters in Hydra and detected and spectroscopically confirmed 118 objects with total $V$-band luminosities between $M_{\mathrm{V}}=-9.7$ and -13.3 mag and projected distances between 3 and $300 \mathrm{kpc}$. The median projected distance is $44 \mathrm{kpc}$. Only 26 of the 118 stellar objects are located within two HST WFPC2 fields (see Fig. 18). 19 of the 26 objects are EOs and the remaining 7 objects have effective radii between 8 and 10 pc. It is expected that also a large fraction of the remaining $92 \mathrm{ob}-$ jects in the outer halo are EOs. In addition, Fig. 7 of Misgeld et al. (2011) demonstrates that star clusters are not uniformly distributed in the halo. While some halo fields contain several UCDs, other fields have no UCDs at all. Consequently, random samples of small fields in a halo cannot be used to extrapolate to the entire population.

Another example is the giant elliptical galaxy NGC 4365, which was covered by eight HST ACS fields (Blom et al. 2012). Seven HST ACS fields provide a very good coverage of the inner $35 \mathrm{kpc}$ of the galaxy. In their central HST field, Blom et al. (2012) found 681 GC and 30 EO candidates, while the six HST fields surrounding the central field have on average 216 GCs and 26 EOs. The results demonstrate that the number density of compact clusters decreases rapidly toward larger projected distances while the number of EOs per HST field is almost constant. In addition, Blom et al. (2012) searched for star clusters outside the HST fields with ground based telescopes and concluded that the cluster system extends out to projected radii of about $135 \mathrm{kpc}$ and contains about three times the number of clusters as the HST fields. Consequently, there are several hundreds of EOs expected in the halo of NGC 4365 outside the HST fields.

The three examples M 51, NGC 4365 and the Hydra Cluster are typical for the entire sample of galaxies with EOs. The vast majority of the studied galaxies have a reasonable coverage of the main stellar body, but a very poor coverage of the halo by
HST observations. Only very distant galaxies like the elliptical galaxy ESO325-G004 in the galaxy cluster Abell S0740 at a Galactic distance of $143 \mathrm{Mpc}$ are well covered (including the halo) by one HST ACS field. However, the pixel size of the HST ACS of $0.05^{\prime \prime}$ corresponds to a linear scale of about $35 \mathrm{pc}$ at this distance, which is too coarse to resolve rather compact EOs.

A considerably larger fraction of the halos of early and latetype galaxies needs to be covered by HST observations to allow for a conclusive view on the spatial distribution of EOs and possible differences between early and late-type galaxies.

\subsection{An EO formation scenario}

Phillipps et al. (2001) interpreted the high-luminosity EOs as a new type of galaxy and reflected this interpretation in the name "ultra-compact dwarf galaxy" (UCD). Bekki et al. (2001, 2003) suggested that UCDs are the remnants of dwarf galaxies which lost their dark matter halo and all stars except for their nucleus. Next to the interpretation as a galaxy, UCDs were also considered as high-mass versions of normal GCs (Mieske et al. 2002), or as merged massive complexes of star clusters (Kroupa 1998; Fellhauer \& Kroupa 2002a; Brüns et al. 2011). In this paper, we will focus on the latter, i.e. the star cluster origin where EOs are the end products of merged star clusters.

A few decades ago "young massive star clusters" (YMCs) were found with globular cluster-like properties. YMCs are found in all types of gas-rich galaxies and constitute a common class of star clusters. The definition of YMCs is rather author dependent. We adopt the definition of Whitmore (2003) who defined "young" as having an age less than $500 \mathrm{Myr}$ and "massive" as having masses ranging from $10^{3} M_{\odot}$ to $10^{8} M_{\odot}$. Individual YMCs were analyzed in detail by Bastian et al. (2006b), Mengel et al. (2008), and Bastian et al. (2009). The combined dataset of the three publications demonstrates that the median size of YMCs in the mass range between $10^{5} M_{\odot}$ and $10^{6} M_{\odot}$ is about 4 pc. YMCs with masses of a few times $10^{7} M_{\odot}$ have only been observed in strong starburst environments like for example in the interacting galaxy NGC 6745 (de Grijs et al. 2003) and the late-stage merger galaxies NGC 7252 (Maraston et al. 2004) and Arp220 (Wilson et al. 2006).

Observations have shown that YMCs are often not isolated, but are part of larger structures called CCs (e.g. Bastian et al. 2006a). The CCs contain few to hundreds of YMCs spanning up to a few hundred parsecs in diameter. The mass of a $\mathrm{CC}$ is the sum of its YMC constituents. Little is known about the detailed distribution of the individual star clusters inside a CC and about their velocity distribution, largely because the existence of CCs had not been realized fully until only very recently. However, the observations show that most CCs have a massive concentration of star clusters in their centers and a couple of isolated star clusters in their vicinity.

Examples for CCs are the knots of the interacting galaxies NGC 4038 and NGC 4039 at a distance of about $20 \mathrm{Mpc}$, aka the Antennae, which have typical masses on the order of $M_{\mathrm{knot}}=$ $10^{6}$ to $10^{7} M_{\odot}$ and sizes of a few hundred parsecs (Whitmore et al. 2005). The knots typically consist of about 30 young massive star clusters with masses greater than $10^{4} M_{\odot}$ and about 60 lower-mass clusters (Whitmore et al. 2010). Whitmore et al. (2005) found that the cluster to cluster velocity dispersion in the knots is small enough to keep them gravitationally bound leading to merging of clusters in the central region of the knots. The collision between the two gas-rich Antennae galaxies triggered the formation of super giant molecular complexes 
with masses up to $9 \times 10^{8} M_{\odot}$ (Wilson et al. 2003). It is expected that these super massive gas clouds will be the birth sites of very massive CCs. Some starburst galaxies like for example Arp 220 at a distance of $77 \mathrm{Mpc}$ host YMCs/CCs as massive as $10^{7} M_{\odot}$ with ages less than $10 \mathrm{Myr}$ (Wilson et al. 2006). Arp 220 also represents two colliding spiral galaxies, but already in the end phase of the merging process. The late-stage merger galaxy NGC 7252 hosts a very massive star cluster (W3) with an age between 300 and 500 Myr. It has a mass of about $8 \times 10^{7} M_{\odot}$ and an effective radius of $R_{\text {eff }}=17.5 \mathrm{pc}$ (Maraston et al. 2004). From its structural parameters W3 may be classified as a young version of a UCD. The young age of $\mathrm{W} 3$ precludes an origin as a remnant nucleus of a stripped dwarf galaxy. W3 may instead have evolved from a CC by merging of its constituent star clusters (Fellhauer \& Kroupa 2005). Another example is the Tadpole galaxy (UGC 10214), which is a disrupted barred spiral galaxy at a distance of about $130 \mathrm{Mpc}$. The galaxy shows a long tidal tail of stars, which hosts also some CCs. The most luminous and largest $\mathrm{CC}$ in the tail has a mass of the order $M_{\mathrm{CC}}=10^{6} M_{\odot}$, an effective radius of $r_{\mathrm{eff}}=160 \mathrm{pc}$, a cutoff radius of about $r_{\text {cut }}=750 \mathrm{pc}$ and it is located at approximately $60 \mathrm{kpc}$ from the center of the galaxy (Tran et al. 2003).

Since galaxy-galaxy mergers are anticipated to have been more common during early cosmological times it is expected that star formation in CCs has been a significant star formation mode during this epoch. Indeed, the preponderance of clumpy galaxies (Elmegreen 2007, and references therein) indicates that early gas-rich galaxies went through an epoch of profuse CC formation. Bournaud et al. (2008a) performed highresolution modeling of a galaxy interaction that lead to a merger remnant comparable to an elliptical galaxy. In these models, super star clusters with masses of a few $10^{7} M_{\odot}$ and sizes up to about $150 \mathrm{pc}$ formed in the halo of this merger remnant. Bournaud et al. (2008b) demonstrated that these star clusters are gravitationally stable.

The dynamical evolution of CCs with a large range of initial configurations and on various orbits was studied in a number of publications (e.g. Kroupa 1998; Fellhauer \& Kroupa 2002a,b, 2005; Bekki et al. 2004; Brüns et al. 2009, 2011; Brüns \& Kroupa 2011). These studies demonstrated that the merging star cluster scenario provides a mechanism for the formation of EOs.

In a previous paper (Brüns et al. 2011), we varied sizes and masses of the modeled CCs covering a matrix of $5 \times 6$ values with effective radii between 10-160 pc and CC masses between $10^{5.5}-10^{8} M_{\odot}$. These models were placed on eccentric orbits between 20 and $60 \mathrm{kpc}$ and were simulated over a period of $5 \mathrm{Gyr}^{2}$. In addition, models with CCs having effective radii of $160 \mathrm{pc}$ were placed on a circular orbit at a distance of $60 \mathrm{kpc}$. Figure 19 shows the location of the initial CCs having $r_{\text {eff }}=160 \mathrm{pc}$ and the corresponding modeled merger objects on the eccentric and the circular orbit after 5 Gyr of evolution. Due to the merging process and the external tidal field, the final masses and effective radii of the merger objects are considerably lower than those of the initial CCs. The comparison between the eccentric and the circular orbits demonstrates that the tidal field plays a crucial role for the final parameters of the merger objects.

Figure 20 shows effective radii and estimated total $V$-band luminosities of the models of Brüns et al. (2011) using a massto-light ratio of 3 and the parameters of the 813 EOs as given in Fig. 1. Figure 20 demonstrates that the observed and simulated

\footnotetext{
2 Brüns \& Kroupa (2011) demonstrated that the structural parameters of the merger objects change only marginally after the merging process is concluded after a few Gyr.
}

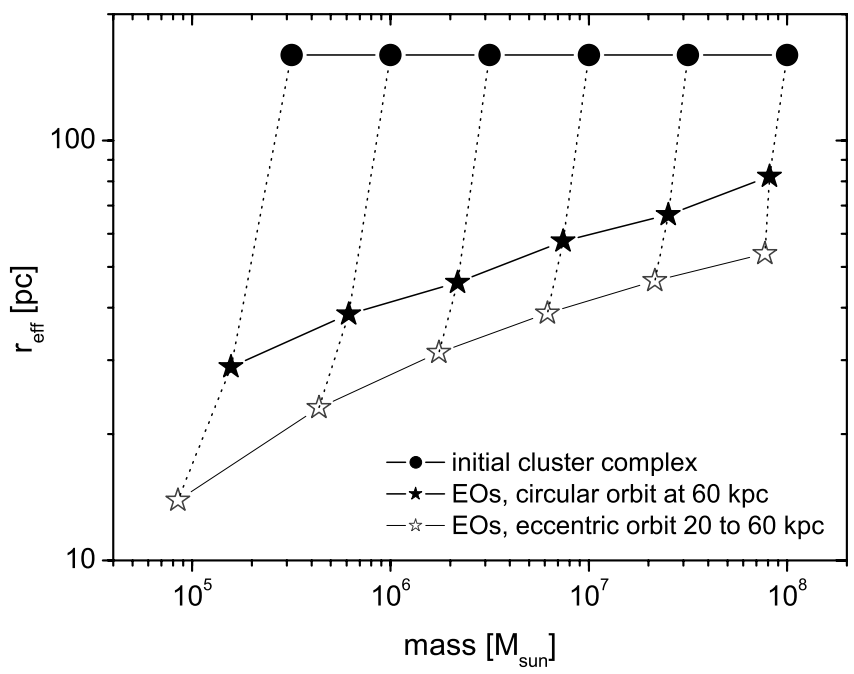

Fig. 19. Diagram of $r_{\text {eff }}$ as a function of mass for the modeled merger objects on a circular orbit at $60 \mathrm{kpc}$ (black stars) and on an eccentric orbit between 20 and $60 \mathrm{kpc}$ (open stars) after a dynamical evolution of $5 \mathrm{Gyr}$ (see Brüns et al. 2011, for details). For comparison, the parameters of the initial CCs (black circles) are included. The initial CCs are connected with the corresponding merger objects by dotted lines.

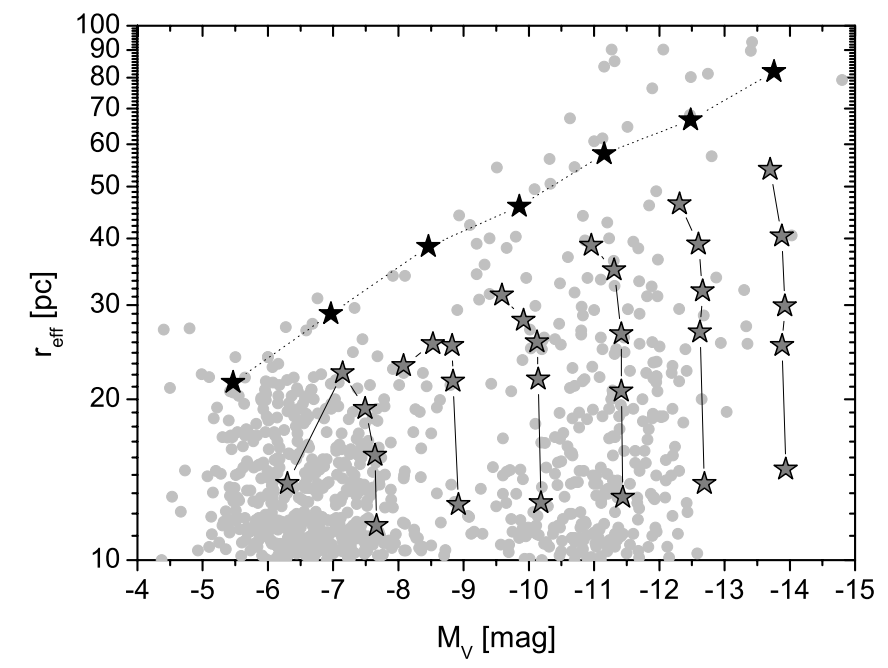

Fig. 20. Diagram of $r_{\text {eff }}$ as a function of the total luminosity of the observed EOs (grey circles) and the modeled merger objects (stars) from Brüns et al. (2011). Merger objects on eccentric orbits between 20 and $60 \mathrm{kpc}$ are plotted in dark grey. Objects with the same initial CC mass but different initial CC effective radii between 10 and $160 \mathrm{pc}$ are connected by black lines. Merger objects on a circular orbit at $60 \mathrm{kpc}$ and an initial effective radius of the CC of $160 \mathrm{pc}$ are plotted as black stars connected by dotted lines.

EOs cover the same parameter space and show the same trend of increasing effective radii with increasing luminosity.

The merged cluster scenario also helps to understand the difference between the mass function of YMCs, which is a powerlaw with a slope of -2 and the GC/EO luminosity functions, which have a bell-shaped function as given in Eq. (2). Most publications focused on the destruction of low-mass clusters (for a recent review see Portegies Zwart et al. 2010, and references therein). The merged cluster scenario predicts that dozens of low-mass YMCs merge to form one EO. As EOs add approximately $10 \%$ to the number of GCs, it is expected that the total number of low-mass clusters needed to build up the EOs is larger than the actual number of remaining GCs. Consequently, 
the merged cluster scenario predicts that a fair fraction of the low-mass YMCs evolve via merging into EOs modifying the original power-law luminosity function.

YMCs and CCs with masses below $M=10^{6} M_{\odot}$ have been observed in all types of galaxies containing sufficient amounts of gas to form star clusters, while high-mass YMCs and CCs $\left(M \gtrsim\right.$ few $\left.\times 10^{6} M_{\odot}\right)$ were so far only observed in major merger events like the Antennae, Arp 220, or NGC 7252, which may be in the process of becoming elliptical galaxies. This explains the different EO luminosity functions for late-type and early-type galaxies: only the major interactions that create the early-type galaxies build CCs massive enough to form the EOs considerably brighter than about $M_{\mathrm{V}}=-10$ mag.

The evolution of low-mass EOs in a weak gravitational environment has also been studied by Hurley \& Mackey (2010), who performed direct $N$-body models of extended low-mass star clusters incorporating a stellar mass function and stellar evolution. Internal evolution processes of the star clusters lead to considerably larger effective radii compared to the initial values. They concluded that extended star clusters with an initial mass of $5.8 \times 10^{4} M_{\odot}$ are sufficiently stable to survive a Hubble-time in a weak gravitational field environment.

Murray (2009) addressed the question why compact star clusters are absent in the high-mass regime, i.e. at masses larger than few $10^{6} M_{\odot}$. Murray (2009) modeled proto-clusters and found that high-mass clusters become optical thick to infra-red radiation leading to a modified initial mass function of the cluster stars, to a larger mass-to-light ratio, and to considerably larger sizes of the proto-clusters. While the idea of optically thick proto-clusters explains the absence of compact clusters in the high-mass regime, it cannot explain the existence of EOs over the large observed mass-range.

An alternative formation scenario for high-mass EOs is the galaxy threshing scenario, where nucleated dwarf galaxies loose, during a heavy interaction with a larger galaxy, almost all stars of the main body except for the nucleus (Bekki et al. 2001). This formation scenario connects EOs with compact elliptical galaxies like M 32, as this rare species of compact galaxy is also expected to be the end-product of an interaction that stripped the bigger part of a formerly larger galaxy. Bekki et al. (2003) performed numerical simulations to demonstrate that nucleated dwarf galaxies can indeed evolve into EOs if they are on a highly eccentric orbit. However, these studies on the threshing scenario focussed on the UCD regime. So far, it was not demonstrated that the threshing scenario is able to reproduce also low-mass EOs.

According to Bekki et al. (2003), a two-component "corehalo" surface brightness profile is a major prediction of the galaxy threshing scenario, where the core is the former nucleus and the halo is the remnant of the main body of the former galaxy.

Three of the four most luminous confirmed EOs, i.e. VUCD7, UCD3, and M59cO, show a clear two-component or core-halo surface brightness profile. Following the results of Bekki et al. (2003), this kind of structure was used as evidence for a galactic origin of UCDs (e.g. Haşegan et al. 2005; Chilingarian \& Mamon 2008; Norris \& Kannappan 2011). While UCD3 is largely overlapping with a background spiral galaxy and $\mathrm{M} 59 \mathrm{cO}$ has a projected distance to M59 of only $9 \mathrm{kpc}$, the most extended and most luminous of the three objects, VUCD7, is located about $83 \mathrm{kpc}$ from M 87 and has neither disturbing foreground nor background objects in its vicinity. Figure 21 shows an HST ACS high-resolution image in the

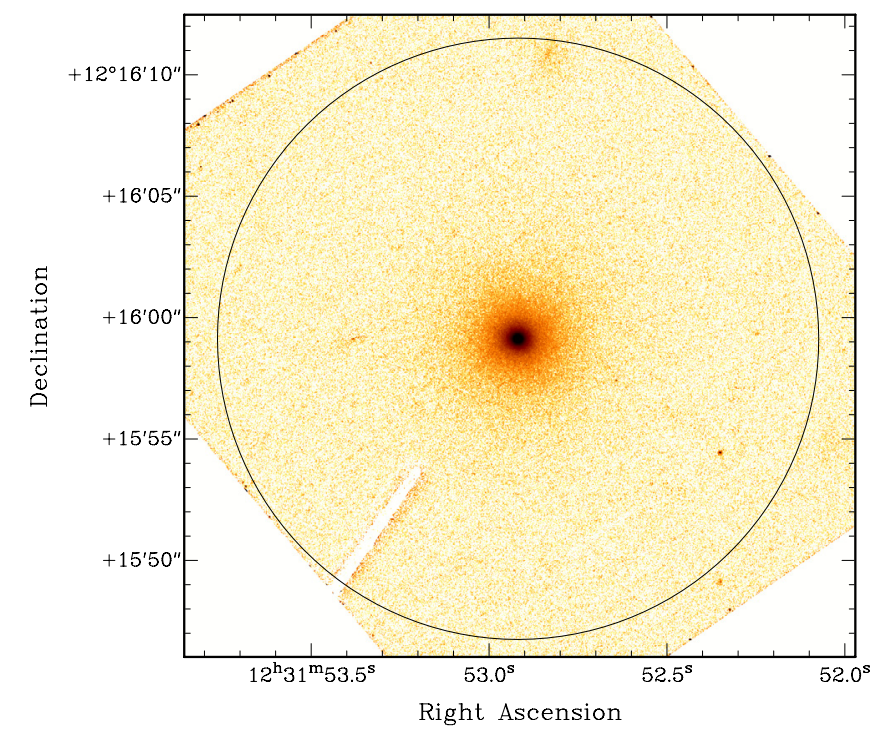

Fig. 21. High resolution HST ACS image of VUCD7 in the $F 606 \mathrm{~W}$ filter. The black circle has a projected radius of $1 \mathrm{kpc}$ indicating the extent of the surface brightness profile shown in Fig. 22.

$F 605 \mathrm{~W}$ band $^{3}$. The HST data allow to compile a surface brightness profile out to large projected radii of about $1 \mathrm{kpc}$.

Figure 22 shows the surface brightness profile of VUCD7 compiled from the archival HST ACS data presented in Fig. 21 using a median filter for the radial bins to exclude faint emission from possible foreground or background objects. The observed profile of VUCD7 shows a clear two-component structure. Whereas most of the models from Brüns et al. (2011) show a single component profile, the surface brightness profiles of the most massive and most extended models on a circular $60 \mathrm{kpc}$ orbit, which have a comparable mass and effective radius as VUCD7, show a two-component profile. Figure 22 demonstrates that the surface brightness profile of model "CC_56_60-kpc" from Brüns et al. (2011) shows a very similar two-component structure as the observed VUCD7. Consequently, a two-component "core-halo" surface brightness profile cannot be used as evidence for the stripping scenario as also the merged cluster scenario explains this specific structure of the most extreme UCDs.

In addition, Brüns et al. (2009) discussed the origin of the EOs, or faint fuzzies, associated with the disk of NGC 1023 in the context of merged cluster scenario and demonstrated that the observed structural parameters of the faint fuzzies are in excellent agreement with the modeled merger objects.

In conclusion, the merged star cluster origin for EOs explains the existence of EOs over the entire observed range of luminosities of ECs and UCDs, the trends in the structural parameters, and the differences between early and late-type galaxies at the high-luminosity end.

\section{Summary and conclusions}

We searched the available literature to compile the largest possible catalog of star clusters with effective radii larger than $10 \mathrm{pc}$. As there is no clear distinction between ECs and UCDs, both types of objects are called extended stellar objects - abbreviated "EOs" - in this paper.

3 The HST ACS image of VUCD7 was taken from the Hubble Legacy Archive, see http://hla.stsci.edu/hlaview . html 


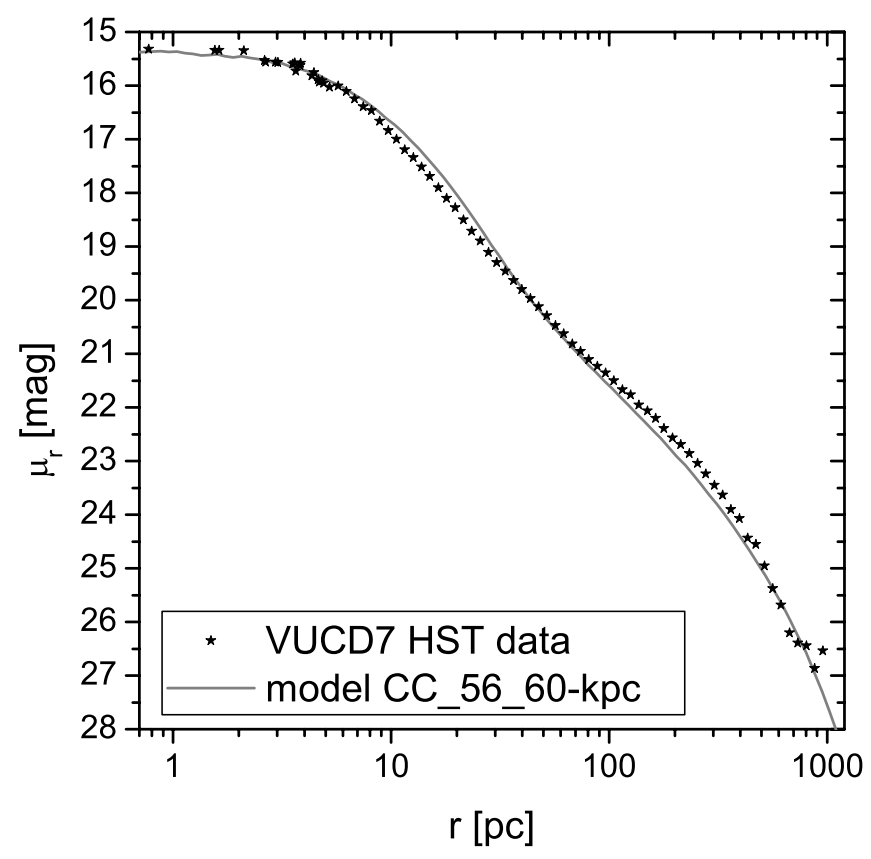

Fig. 22. Surface brightness profile compiled from archival HST ACS data (black stars) of the EO VUCD7 and the surface brightness profile of model CC_56_60-kpc (grey line) from Brüns et al. (2011) using a mass-to-light ratio of 2.8. The model and the observed EO show a very similar two-component or "core-halo" structure.

In total, we compiled a catalog of 813 EOs of which 171 were found associated with late-type galaxies and 642 EOs associated with early-type galaxies. The main results presented in this paper are

1. EOs cover a luminosity range from about $M_{\mathrm{V}}=-4$ to -14 mag. However, almost all EOs brighter than $M_{\mathrm{V}}=$ -10 mag are associated with giant elliptical galaxies.

2. At all values of $M_{\mathrm{V}}$ extended objects are found with effective radii between $10 \mathrm{pc}$ and an upper size limit, which shows a clear trend: the more luminous the object the larger is the upper size limit. This upper limit increases from about $30 \mathrm{pc}$ at $M_{\mathrm{V}}=-5$ mag to about $100 \mathrm{pc}$ at $M_{\mathrm{V}}=-14 \mathrm{mag}$.

3. The 175 confirmed EOs cover the same region in the $r_{\mathrm{eff}} \mathrm{vs}$. $M_{\mathrm{V}}$ space and show the same trends of increasing size with increasing luminosities as the 638 candidate EOs.

4. For all luminosities, the majority of EOs have effective radii which are only slightly larger than $10 \mathrm{pc}$. The median effective radius of EOs in late-type and early-type galaxies is $13.2 \mathrm{pc}$ and $14.2 \mathrm{pc}$, respectively.

5. The effective radii are increasing with increasing total luminosity of the host galaxy.

6. For late-type galaxies there is no trend of the EO luminosities with the luminosity of the host galaxies, while for earlytype galaxies there is a trend that the most luminous EOs are found associated with the most luminous galaxies.

7. EOs and GCs form a coherent structure in the $r_{\mathrm{eff}}$ vs. $M_{\mathrm{V}}$ parameter space, which is well separated from the distribution of early type dwarf galaxies, except for the rare species of compact elliptical galaxies. Especially at the low-luminosity end considerably deeper observations are needed to answer the question, whether the prominent gap between ECs and $\mathrm{dSph}$ galaxies is real.

8. For both EOs associated with early and late-type galaxies, the EO luminosity functions peak at about $-6.5 \mathrm{mag}$, which is roughly one magnitude fainter than the turnover of the GC luminosity function. The turnover luminosities decrease continuously between compact GCs and EOs for increasing effective radii. Considering the very low surface brightness of faint and extended EOs, a fair fraction of very extended and faint EOs is most likely below the detection limit of extragalactic surveys. The true turnover of the EO luminosity function might therefore be at even lower luminosities.

We discussed an EO formation scenario on the basis of a star cluster origin which explains the existence of EOs, the trends of the structural parameters and the differences between early and late-type galaxies over the entire range of luminosities of ECs and UCDs. In addition, a core-halo surface brightness profile, as observed for some massive EOs like VUCD7, can be reproduced in the merged CC formation scenario.

While this study presents the to day largest catalog of EOs, it suffers from substantial incompleteness, predominantly with respect to the coverage of the galactic halos, but also with respect to varying detection limits and the fairly low number of confirmed objects. Larger and more complete data-sets and additional information on parameters like metallicities, mass-to-light ratios and age-estimates are necessary to draw final conclusions on the origin of EOs, which in turn has the potential to shed light on the cosmologically important phase of galaxy formation.

Acknowledgements. This work was supported by the German Deutsche Forschungsgemeinschaft, DFG project number KR 1635/29-1. We thank J. P. Madrid and S. Larsen for providing additional data on EOs not directly accessable from Madrid (2011) and Larsen \& Brodie (2000). We thank the anonymous referee for his helpful comments, which lead to an improved paper.

\section{References}

Bastian, N., Emsellem, E., Kissler-Patig, M., \& Maraston, C. 2006a, A\&A, 445, 471

Bastian, N., Saglia, R. P., Goudfrooij, P., et al. 2006b, A\&A, 448, 881

Bastian, N., Trancho, G., Konstantopoulos, I. S., \& Miller, B. W. 2009, ApJ, 701, 607

Bekki, K., Couch, W. J., \& Drinkwater, M. J. 2001, ApJ, 552, L105

Bekki, K., Couch, W. J., Drinkwater, M. J., \& Shioya, Y. 2003, MNRAS, 344, 399

Bekki, K., Couch, W. J., Drinkwater, M. J., \& Shioya, Y. 2004, ApJ, 610, L13

Bell, E. F., Slater, C. T., \& Martin, N. F. 2011, ApJ, 742, L15

Belokurov, V., Walker, M. G., Evans, N. W., et al. 2010, ApJ, 712, L103

Blakeslee, J. P., \& Barber DeGraaff, R. 2008, AJ, 136, 2295

Blom C., Spitler, L. R., \& Forbes, D. A. 2012, MNRAS, 420, 37

Bournaud, F., Bois, M., Emsellem, E., \& Duc, P.-A. 2008a, Astron. Nachr., 329, 1025

Bournaud, F., Duc, P.-A., \& Emsellem, E. 2008b, MNRAS, 389, L8

Brasseur, C. M., Martin, N. F., Macciò, A. V., Rix, H.-W., \& Kang, X. 2011, ApJ, 743, 179

Brodie, J. P., \& Larsen, S. S. 2002, AJ, 124, 1410

Brodie, J. P., \& Strader, J. 2006, ARA\&A, 44, 193

Brodie, J. P., Romanowsky, A. J., Strader, J., \& Forbes, D. A. 2011, AJ, 142, 199

Brüns, R. C., \& Kroupa, P. 2011, ApJ, 729, 69

Brüns, R. C., Kroupa, P., \& Fellhauer, M. 2009, ApJ, 702, 1268

Brüns, R. C., Kroupa, P., Fellhauer, M., Metz, M., \& Assmann, P. 2011, A\&A, 529, A138

Cantiello, M., Brocato, E., \& Blakeslee, J. P. 2009, A\&A, 503, 87

Cappellari, M., Bacon, R., Bureau, M., et al. 2006, MNRAS, 366, 1126

Chandar, R., Whitmore, B., \& Lee, M. G. 2004, ApJ, 611, 220

Chattopadhyay, A. K., Chattopadhyay, T., Davoust, E., Mondal, S., \& Sharina, M. 2009, ApJ, 705, 1533

Chiboucas, K., Tully, R. B., Marzke, R. O., et al. 2011, ApJ, 737, 86

Chies-Santos, A. L., Pastoriza, M. G., Santiago, B. X., \& Forbes, D. A. 2006, A\&A, 455, 453

Chies-Santos, A. L., Santiago, B. X., \& Pastoriza, M. G. 2007, A\&A, 467, 1003

Chies-Santos, A. L., Larsen, S. S., Wehner, E. M., et al. 2011, A\&A, 525, A19

Chilingarian, I. V., \& Mamon, G. A. 2008, MNRAS, 385, L83

Chilingarian, I. V., Mieske, S., Hilker, M., \& Infante, L. 2011, MNRAS, 412, 1627 
Cockcroft, R., Harris, W. E., Ferguson, A. M. N., et al. 2011, ApJ, 730, 112 Dabringhausen, J., Hilker, M., \& Kroupa, P. 2008, MNRAS, 386, 864 Dabringhausen, J., Kroupa, P., \& Baumgardt, H. 2009, MNRAS, 394, 1529

Da Costa, G. S., Grebel, E. K., Jerjen, H., Rejkuba, M., \& Sharina, M. E. 2009, AJ, 137, 4361

Da Rocha, C., Mieske, S., Georgiev, I. Y., et al. 2011, A\&A, 525, A86

DeGraaff, R. B., Blakeslee, J. P., Meurer, G. R., \& Putman, M. E. 2007, ApJ, 671,1624

de Grijs, R., Anders, P., Bastian, N., et al. 2003, MNRAS, 343, 1285

Drinkwater, M. J., Jones, J. B., Gregg, M. D., \& Phillipps, S. 2000, PASA, 17, 227

Elmegreen, D. M. 2007, in IAU Symp. 235, eds. F. Combes, \& J. Palous, 376

Evstigneeva, E. A., Gregg, M. D., Drinkwater, M. J., \& Hilker, M. 2007, AJ, 133,1722

Evstigneeva, E. A., Drinkwater, M. J., Peng, C. Y., et al. 2008, AJ, 136, 461

Fellhauer, M., \& Kroupa, P. 2002a, MNRAS, 330, 642

Fellhauer, M., \& Kroupa, P. 2002b, AJ, 124, 2006

Fellhauer, M., \& Kroupa, P. 2005, MNRAS, 359, 223

Forbes, D. A., Lasky, P., Graham, A. W., \& Spitler, L. 2008, MNRAS, 389, 1924

Geha, M., van der Marel, R. P., Guhathakurta, P., et al. 2010, ApJ, 711, 361

Georgiev, I. Y., Puzia, T. H., Hilker, M., \& Goudfrooij, P. 2009, MNRAS, 392, 879

Gilmore, G., Wilkinson, M. I., Wyse, R. F. G., et al. 2007, ApJ, 663, 948

Goudfrooij, P. 2012, ApJ, 750, 140

Gómez, M., Geisler, D., Harris, W. E., et al. 2006, A\&A, 447, 877

Harris, W. E. 1996, AJ, 112, 1487

Harris, W. E., Mouhcine, M., Rejkuba, M., \& Ibata, R. 2009, MNRAS, 395, 436

Hassegan, M., Jordán, A., Côté, P., et al. 2005, ApJ, 627, 203

Hau, G. K. T., Spitler, L. R., Forbes, D. A., et al. 2009, MNRAS, 394, L97

Hilker, M., Infante, L., Vieira, G., Kissler-Patig, M., \& Richtler, T. 1999, A\&AS, 134,75

Hilker, M., Baumgardt, H., Infante, L., et al. 2007, A\&A, 463, 119

Hurley, J. R., \& Mackey, A. D. 2010, MNRAS, 408, 2353

Huxor, A. P., Tanvir, N. R., Irwin, M. J., et al. 2005, MNRAS, 360, 1007

Huxor, A. P., Tanvir, N. R., Ferguson, A. M. N., et al. 2008, MNRAS, 385, 1989

Huxor, A. P., Ferguson, A. M. N., Barker, M. K., et al. 2009, ApJ, 698, L77

Huxor, A. P., Ferguson, A. M. N., Tanvir, N. R., et al. 2011a, MNRAS, 414, 770

Huxor, A. P., Phillipps, S., Price, J., \& Harniman, R. 2011b, MNRAS, 414, 3557

Hwang, N., \& Lee, M. G. 2008, AJ, 135, 1567

Hwang, N., Lee, M. G., Lee, J. C., et al. 2011, ApJ, 738, 58

Jordán, A., Côté, P., Blakeslee, J. P., et al. 2005, ApJ, 634, 1002

Kroupa, P. 1998, MNRAS, 300, 200

Larsen, S. S., \& Brodie, J. P. 2000, AJ, 120, 2938

Larsen, S. S., Forbes, D. A., \& Brodie, J. P. 2001, MNRAS, 327, 1116

Mackey, A. D., \& Gilmore, G. F. 2004, MNRAS, 352, 153

Madrid, J. P. 2011, ApJ, 737, L13

Madrid, J. P., Graham, A. W., Harris, W. E., et al. 2010, ApJ, 722, 1707

Maraston, C., Bastian, N., Saglia, R. P., et al. 2004, A\&A, 416, 467

Martin, N. F., de Jong, J. T. A., \& Rix, H.-W. 2008, ApJ, 684, 1075

Masters, K. L., Jordán, A., Côté, P., et al. 2010, ApJ, 715, 1419

McConnachie, A. W. 2012, AJ, 144, 4

McConnachie, A. W., \& Irwin, M. J. 2006, MNRAS, 365, 1263

McLaughlin, D. E., \& van der Marel, R. P. 2005, ApJS, 161, 304
McLaughlin, D. E., Barmby, P., Harris, W. E., Forbes, D. A., \& Harris, G. L. H. 2008, MNRAS, 384, 563

Mengel, S., Lehnert, M. D., Thatte, N. A., et al. 2008, A\&A, 489, 1091

Mieske, S., \& Kroupa, P. 2008, ApJ, 677, 276

Mieske, S., Hilker, M., \& Infante, L. 2002, A\&A, 383, 823

Mieske, S., Infante, L., Hilker, M., et al. 2005, A\&A, 430, L25

Mieske, S., Hilker, M., Jordán, A., Infante, L., \& Kissler-Patig, M. 2007, A\&A, 472,111

Mieske, S., Hilker, M., Jordán, A., et al. 2008, A\&A, 487, 921

Mieske, S., Hilker, M., \& Misgeld, I. 2012, A\&A, 537, A3

Misgeld, I., \& Hilker, M. 2011, MNRAS, 414, 3699

Misgeld, I., Mieske, S., Hilker, M., et al. 2011, A\&A, 531, A4

Mouhcine, M., Harris, W. E., Ibata, R., \& Rejkuba, M. 2010, MNRAS, 404, 1157

Murray, M. 2009, ApJ, 691, 946

Nantais, J. B., Huchra, J. P., Zezas, A., Gazeas, K., \& Strader, J. 2011, AJ, 142, 183

Norris, M. A., \& Kannappan, S. J. 2011, MNRAS, 414, 739

Peacock, M. B., Maccarone, T. J., Waters, C. Z., et al. 2009, MNRAS, 392, L55

Pellerin, A., Meurer, G. R., Bekki, K., et al. 2010, AJ, 139, 1369

Peng, E. W., Côté, P., Jordán, A., et al. 2006, ApJ, 639, 838

Penny, S. J., Forbes, D. A., \& Conselice, C. J. 2012, MNRAS, 422, 885

Phillipps, S., Drinkwater, M. J., Gregg, M. D., \& Jones, J. B. 2001, ApJ, 560, 201

Portegies Zwart, S. F., McMillan, S. L. W., \& Gieles, M. 2010, ARA\&A, 48, 431

Price, J., Phillipps, S., Huxor, A., et al. 2009, MNRAS, 397, 1816

Rejkuba, M. 2012, Ap\&SS, 341, 195

Richtler, T., Dirsch, B., Larsen, S., Hilker, M., \& Infante, L. 2005, A\&A, 439, 533

Salinas, R., Jilkova, L., Carraro, G., Catelan, M., \& Amigo, P. 2012. MNRAS, 421,960

Secker, J. 1992, AJ, 104, 1472

Sharina, M. E., Puzia, T. H., \& Makarov, D. I. 2005, A\&A, 442, 85

Sharina, M. E., Karachentsev, I. D., Dolphin, A. E., et al. 2008, MNRAS, 384, 1544

Slater, C. T., Bell, E. F., \& Martin, N. F. 2011, ApJ, 742, L14

Smith Castelli, A. V., Faifer, F. R., Richtler, T., \& Bassino, L. P. 2008, MNRAS, 391,685

Smith Castelli, A. V., Cellone, S. A., Faifer, F. R., et al. 2012, MNRAS, 419, 2472

Stonkutè, R., Vansevičius, V., Arimoto, N., et al. 2008, AJ, 135, 1482

Strader, J., Seth, A. C., \& Caldwell, N. 2012, AJ, 143, 52

Taylor, M. A., Puzia, T. H., Harris, G. L., et al. 2010, ApJ, 712, 1191

Tran, H. D., Sirianni, M., Ford, H. C., et al. 2003, ApJ, 585, 750

van den Bergh, S. 2006, AJ, 131, 304

van den Bergh, S., \& Mackey, A. D. 2004, MNRAS, 354, 713

Whitmore, B. C. 2003, in A Decade of Hubble Space Telescope Science, eds. M. Livio, K. Noll, \& M. Stiavelli, 153

Whitmore, B. C., Gilmore, D., Leitherer, C., et al. 2005, AJ, 130, 2104

Whitmore, B. C., Chandar, R., Schweizer, F., et al. 2010, AJ, 140, 75

Wilson, C. D., Scoville, N., Madden, S. C., \& Charmandaris, V. 2003, ApJ, 599, 1049

Wilson, C. D., Harris, W. E., Longden, R., \& Scoville, N. Z. 2006, ApJ, 641, 763 
R. C. Brüns and P. Kroupa: A catalog of ECs and UCDs

Table 2. Catalog of the 65 galaxies containing EOs.

\begin{tabular}{|c|c|c|c|c|c|c|c|c|c|}
\hline Galaxy & Type & $\begin{array}{l}M_{\mathrm{V}, \mathrm{gal}} \\
(\mathrm{mag})\end{array}$ & $\begin{array}{r}D_{\text {gal }} \\
(\mathrm{kpc}) \\
\end{array}$ & $\begin{array}{c}\text { Pix }_{\text {HST }} \\
(\mathrm{pc})\end{array}$ & $N_{\mathrm{EO}}$ & $\begin{array}{c}M_{\mathrm{V}, \mathrm{EO}, \min } \\
(\mathrm{mag})\end{array}$ & $\begin{array}{c}M_{\mathrm{V}, \mathrm{EO}, \max } \\
(\mathrm{mag})\end{array}$ & $N_{\mathrm{GC}}$ & Ref. \\
\hline Milky Way & LT & -20.5 & 0 & & 11 & -4.73 & -9.42 & 142 & 1 \\
\hline SAGdSph & LT & -13.8 & 27 & & 2 & -5.41 & -5.68 & 2 & 2 \\
\hline LMC & LT & -18.34 & 50 & & 4 & -4.37 & -7.25 & 12 & 3 \\
\hline Fornax & ET & -13.3 & 138 & & 1 & -5.32 & -5.32 & 4 & 3 \\
\hline NGC 6822 & LT & -16 & 470 & 0.1 & 3 & -6.06 & -7.7 & 3 & 4 \\
\hline M 31 & LT & -21.8 & 780 & 0.2 & 20 & -4.4 & -7.68 & 232 & $5 ; 6 ; 7$ \\
\hline M 33 & LT & -19.4 & 870 & 0.2 & 2 & -5.9 & -6.6 & 4 & $8 ; 9$ \\
\hline UGCA 86 & LT & -13.55 & 2728 & 0.7 & 1 & -7.58 & -7.58 & 11 & 10 \\
\hline UGC 8638 & LT & -13.8 & 3285 & 0.8 & 1 & -6.57 & -6.57 & 2 & 10 \\
\hline NGC 247 & LT & -19.4 & 3636 & 0.9 & 2 & -6.59 & -7.42 & 0 & 10 \\
\hline NGC 5128 & ET & -21.4 & 3676 & 0.9 & 26 & -5.2 & -11.17 & 194 & $11 ; 12 ; 13 ; 14 ; 15$ \\
\hline M 81 & LT & -21.2 & 3692 & 0.9 & 44 & -4.53 & -8.59 & 369 & 16 \\
\hline NGC 4449 & LT & -18.28 & 3693 & 0.9 & 7 & -5.35 & -7.14 & 99 & 17 \\
\hline IKN & ET & -11.5 & 3680 & 0.9 & 1 & -6.76 & -6.76 & 4 & 10 \\
\hline NGC 5237 & LT & -15.7 & 3794 & 0.9 & 1 & -6.85 & -6.85 & 2 & 10 \\
\hline ESO269-58 & LT & -16.3 & 3825 & 0.9 & 2 & -6.62 & -6.86 & 6 & 10 \\
\hline UGC 7605 & LT & -13.8 & 4177 & 1 & 1 & -6.44 & -6.44 & 0 & $18 ; 19$ \\
\hline Scl-dE1 & ET & -11.1 & 4300 & 1 & 1 & -6.7 & -6.7 & 0 & 20 \\
\hline KK 065 & LT & -13.32 & 4510 & 1.1 & 1 & -6.75 & -6.75 & 0 & $18 ; 19$ \\
\hline NGC 784 & LT & -17.6 & 4560 & 1.1 & 2 & -6.4 & -6.62 & 5 & 10 \\
\hline M 83 & LT & -21 & 4659 & 1.1 & 1 & -8.24 & -8.24 & 20 & 21 \\
\hline NGC 4605 & LT & -18.5 & 4730 & 1.1 & 2 & -6.34 & -8.26 & 9 & 10 \\
\hline UGC 3974 & LT & -15.2 & 4897 & 1.2 & 1 & -8.72 & -8.72 & 4 & 10 \\
\hline UGC 3755 & LT & -15 & 5166 & 1.3 & 2 & -6.09 & -7.54 & 30 & $18 ; 19$ \\
\hline KK 112 & LT & -12.28 & 5220 & 1.3 & 2 & -6.21 & -6.77 & 1 & $18 ; 19$ \\
\hline NGC 1311 & LT & -16.3 & 5252 & 1.3 & 1 & -7.33 & -7.33 & 5 & 10 \\
\hline UGC 4115 & LT & -14.12 & 5508 & 1.3 & 1 & -6 & -6 & 2 & $18 ; 19$ \\
\hline M 51 & LT & -21.4 & 8031 & 1.9 & 21 & -6.91 & -8.86 & 2203 & 22 \\
\hline M 104 & LT & -22.45 & 9000 & 2.2 & 10 & -6.05 & -12.3 & 184 & $23 ; 24$ \\
\hline NGC 891 & LT & -21.2 & 9700 & 2.4 & 6 & -5.16 & -7.3 & 37 & 25 \\
\hline KK 84 & ET & -14.4 & 10069 & 2.4 & 6 & -6.64 & -9.68 & 1 & $18 ; 19$ \\
\hline NGC 1023 & ET & -21.2 & 11791 & 2.9 & 15 & -6.02 & -7.13 & 14 & 26 \\
\hline NGC 4546 & ET & -20.9 & 13060 & 3.2 & 1 & -12.94 & -12.94 & 0 & 27 \\
\hline NGC 4660 & ET & -19.69 & 14875 & 3.6 & 1 & -8.34 & -8.34 & 50 & 28 \\
\hline IC 3652 & ET & -18.7 & 14960 & 3.6 & 1 & -11.95 & -11.95 & 0 & 29 \\
\hline NGC 4278 & ET & -20.78 & 15154 & 3.7 & 1 & -9.93 & -9.93 & 66 & 28 \\
\hline NGC 4486B & ET & -17.64 & 15450 & 3.7 & 1 & -11.98 & -11.98 & 0 & 29 \\
\hline M 89 & ET & -21.32 & 15574 & 3.8 & 3 & -10.44 & -11.6 & 104 & $28 ; 29$ \\
\hline M 59 & ET & -21.38 & 15821 & 3.8 & 1 & -13.3 & -13.3 & 0 & 30 \\
\hline M 49 & ET & -22.63 & 16052 & 3.9 & 1 & -10.47 & -10.47 & 0 & 29 \\
\hline M 86 & ET & -22.21 & 16321 & 4 & 2 & -9.08 & -9.66 & 74 & 28 \\
\hline NGC 4476 & ET & -18.97 & 16450 & 4 & 1 & -10.89 & -10.89 & 0 & 29 \\
\hline M 87 & ET & -22.54 & 16675 & 4 & 51 & -8.1 & -13.42 & 301 & $28 ; 29 ; 31 ; 32$ \\
\hline M 85 & ET & -21.98 & 17382 & 4.2 & 4 & -8.5 & -11.46 & 55 & $28 ; 29$ \\
\hline M 84 & ET & -22.12 & 17422 & 4.2 & 1 & -9.75 & -9.75 & 92 & 28 \\
\hline NGC 1380 & ET & -21.3 & 18221 & 4.4 & 13 & -5.14 & -9.45 & 174 & 34 \\
\hline NGC 1399 & ET & -21.88 & 18950 & 4.6 & 18 & -10.02 & -13.4 & 18 & $31 ; 33 ; 35 ; 36 ; 37 ; 38$ \\
\hline NGC 1533 & ET & -20.7 & 19400 & 4.7 & 3 & -7.04 & -7.52 & 136 & 39 \\
\hline NGC 3923 & ET & -21.9 & 21280 & 5.2 & 3 & -11.29 & -12.43 & 0 & 27 \\
\hline NGC 1316 & ET & -22.91 & 21900 & 5.3 & 45 & -5.65 & -9.26 & 433 & 40 \\
\hline NGC 4365 & ET & -22.13 & 23100 & 5.6 & 217 & -4.66 & -11.84 & 2038 & 41 \\
\hline NGC 5846 & ET & -22.18 & 26709 & 6.5 & 13 & -6.89 & -9.32 & 41 & 42 \\
\hline NGC 3370 & LT & -20 & 27376 & 6.6 & 22 & -5.51 & -7.61 & 255 & 43 \\
\hline NGC 1199 & ET & -21.25 & 33100 & 8 & 2 & -11.97 & -12.15 & 8 & 44 \\
\hline NGC 4696 & ET & -22.81 & 37582 & 9.1 & 2 & -11.1 & -11.52 & 6 & 45 \\
\hline NGC 3311 & ET & -22.2 & 47200 & 11.4 & 19 & -9.29 & -13.35 & 7 & 46 \\
\hline NGC 1275 & ET & -22.72 & 71000 & 17.2 & 84 & -9.98 & -13.33 & 0 & 47 \\
\hline IC 4041 & ET & -20.74 & 94400 & 22.9 & 4 & -10.77 & -11.31 & 0 & 48 \\
\hline NGC 4889 & ET & -23.51 & 94400 & 22.9 & 2 & -11.11 & -11.34 & 2 & 48 \\
\hline IC 3998 & ET & -20.43 & 98950 & 24 & 1 & -11.79 & -11.79 & 0 & 48 \\
\hline IC 4030 & ET & -19.6 & 98950 & 24 & 2 & -10.7 & -11.41 & 0 & 48 \\
\hline NGC 4874 & ET & -23.25 & 98950 & 24 & 36 & -9.85 & -14.03 & 2 & $48 ; 49$ \\
\hline NGC 1132 & ET & -22.65 & 99500 & 24.1 & 39 & -9.24 & -14.8 & 11 & 50 \\
\hline NGC 4908 & ET & -21.82 & 101114 & 24.5 & 3 & -11.08 & -12.47 & 0 & 48 \\
\hline ESO325-G004 & ET & -23.2 & 143000 & 34.7 & 15 & -11.34 & -13.51 & 0 & 51 \\
\hline
\end{tabular}

Notes. The columns denote 1) name of the galaxy; 2) type of host galaxy (LT: late-type, ET: early-type); 3) absolute $V$-band luminosity of the galaxy; 4) distance of the galaxy; 5) size of a HST ACS pixel at the distance of the galaxy; 6) number of EOs; 7) minimum absolute $V$-band luminosity of the EO; 8) maximum absolute $V$-band luminosity of the EO; 9) number of GCs in the same publications.

References. As in Table 1. 